\title{
Search for $\mathbf{B}_{s}^{0}-\overline{\mathbf{B}_{s}^{0}}$ oscillations and a measurement of $\mathbf{B}_{d}^{0}-\overline{\mathbf{B}_{d}^{0}}$ oscillations using events with an inclusively reconstructed vertex
}

\author{
DELPHI Collaboration
}

\begin{abstract}
Neutral B meson oscillations in the $\mathrm{B}_{s}^{0}-\overline{\mathrm{B}_{s}^{0}}$ and $\mathrm{B}_{d}^{0}-\overline{\mathrm{B}_{d}^{0}}$ systems were studied using a sample of about 4.0 million hadronic $\mathrm{Z}$ decays recorded by the DELPHI detector between 1992 and 2000. Events with a high transverse momentum lepton were removed and a sample of $770 \mathrm{k}$ events with an inclusively reconstructed vertex was selected.

The mass difference between the two physical states in the $\mathrm{B}_{d}^{0}-\overline{\mathrm{B}_{d}^{0}}$ system was measured to be:

$$
\Delta m_{d}=(0.531 \pm 0.025(\text { stat. }) \pm 0.007(\text { syst. })) \mathrm{ps}^{-1} .
$$
\end{abstract}

The following limit on the width difference of these states was also obtained:

$$
\left|\Delta \Gamma_{\mathrm{B}_{\mathrm{d}}}\right| / \Gamma_{\mathrm{B}_{\mathrm{d}}}<0.18 \text { at } 95 \% \mathrm{CL} \text {. }
$$

As no evidence for $\mathrm{B}_{s}^{0}-\overline{\mathrm{B}_{s}^{0}}$ oscillations was found, a limit on the mass difference of the two physical states was given:

$$
\Delta m_{s}>5.0 \mathrm{ps}^{-1} \text { at } 95 \% \mathrm{CL} \text {. }
$$

The corresponding sensitivity of this analysis is equal to $6.6 \mathrm{ps}^{-1}$. 


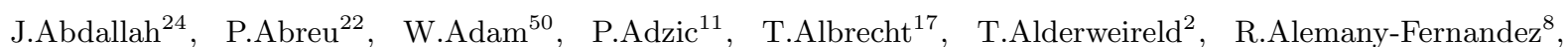
T.Allmendinger ${ }^{17}$, P.P.Allport ${ }^{23}$, U.Amaldi ${ }^{28}$, N.Amapane ${ }^{44}$, S.Amato ${ }^{47}$, E.Anashkin ${ }^{35}$, A.Andreazza ${ }^{27}$, S.Andringa ${ }^{22}$, N.Anjos ${ }^{22}$, P.Antilogus ${ }^{26}$, W-D.Apel ${ }^{17}$, Y.Arnoud ${ }^{14}$, S.Ask ${ }^{25}$, B.Asman ${ }^{43}$, J.E.Augustin ${ }^{24}$, A.Augustinus $^{8}$, P.Baillon ${ }^{8}$, A.Ballestrero ${ }^{45}$, P.Bambade ${ }^{20}$, R.Barbier ${ }^{26}$, D.Bardin ${ }^{16}$, G.Barker ${ }^{17}$, A.Baroncelli $^{38}$, M.Battaglia $^{8}$, M.Baubillier ${ }^{24}$, K-H.Becks ${ }^{52}$, M.Begalli ${ }^{6}$, A.Behrmann ${ }^{52}$, E.Ben-Haim ${ }^{20}$, N.Benekos ${ }^{31}$, A.Benvenuti $^{5}$, C.Berat ${ }^{14}$, M.Berggren ${ }^{24}$, L.Berntzon ${ }^{43}$, D.Bertrand ${ }^{2}$, M.Besancon ${ }^{39}$, N.Besson ${ }^{39}$, D.Bloch ${ }^{9}$, M.Blom $^{30}$, M.Bluj ${ }^{51}$, M.Bonesini ${ }^{28}$, M.Boonekamp ${ }^{39}$, P.S.L.Booth ${ }^{23}$, G.Borisov ${ }^{21}$, O.Botner ${ }^{48}$, B.Bouquet $^{20}$, T.J.V.Bowcock ${ }^{23}$, I.Boyko ${ }^{16}$, M.Bracko ${ }^{42}$, R.Brenner ${ }^{48}$, E.Brodet ${ }^{34}$, P.Bruckman ${ }^{18}$, J.M.Brunet ${ }^{7}$, L.Bugge ${ }^{32}$, P.Buschmann $^{52}$, M.Calvi ${ }^{28}$, T.Camporesi ${ }^{8}$, V.Canale ${ }^{37}$, F.Carena ${ }^{8}$, N.Castro ${ }^{22}$, F.Cavallo ${ }^{5}$, M.Chapkin ${ }^{41}$, Ph.Charpentier $^{8}$, P.Checchia ${ }^{35}$, R.Chierici ${ }^{8}$, P.Chliapnikov ${ }^{41}$, J.Chudoba ${ }^{8}$, S.U.Chung ${ }^{8}$, K.Cieslik ${ }^{18}$, P.Collins ${ }^{8}$, R.Contri ${ }^{13}$, G.Cosme ${ }^{20}$, F.Cossutti ${ }^{46}$, M.J.Costa ${ }^{49}$, B.Crawley ${ }^{1}$, D.Crennell ${ }^{36}$, J.Cuevas ${ }^{33}$, J.D'Hondt ${ }^{2}$, J.Dalmau $^{43}$, T.da Silva ${ }^{47}$, W.Da Silva ${ }^{24}$, G.Della Ricca ${ }^{46}$, A.De Angelis ${ }^{46}$, W.De Boer ${ }^{17}$, C.De Clercq ${ }^{2}$, B.De Lotto ${ }^{46}$, N.De Maria ${ }^{44}$ A.De Min ${ }^{35}$, L.de Paula ${ }^{47}$, L.Di Ciaccio ${ }^{37}$, A.Di Simone ${ }^{38}$, K.Doroba ${ }^{51}$, J.Drees $^{52,8}$, M.Dris ${ }^{31}$, G.Eigen ${ }^{4}$, T.Ekelof ${ }^{48}$, M.Ellert ${ }^{48}$, M.Elsing ${ }^{8}$, M.C.Espirito Santo ${ }^{8}$, G.Fanourakis ${ }^{11}$, D.Fassouliotis ${ }^{11,3}$, M.Feindt ${ }^{17}$, J.Fernandez ${ }^{40}$, A.Ferrer ${ }^{49}$, F.Ferro ${ }^{13}$, U.Flagmeyer ${ }^{52}$, H.Foeth ${ }^{8}$, E.Fokitis ${ }^{31}$, F.Fulda-Quenzer ${ }^{20}$, J.Fuster ${ }^{49}$, M.Gandelman ${ }^{47}$, C.Garcia ${ }^{49}$, Ph.Gavillet $^{8}$, E.Gazis ${ }^{31}$, T.Geralis ${ }^{11}$, R.Gokieli ${ }^{8,51}$, B.Golob ${ }^{42}$, G.Gomez-Ceballos ${ }^{40}$, P.Goncalves ${ }^{22}$, E.Graziani ${ }^{38}$, G.Grosdidier ${ }^{20}$, K.Grzelak ${ }^{51}$, J.Guy ${ }^{36}$, C.Haag $^{17}$, A.Hallgren $^{48}$, K.Hamacher ${ }^{52}$, K.Hamilton ${ }^{34}$, J.Hansen ${ }^{32}$, S.Haug ${ }^{32}$, F.Hauler ${ }^{17}$, V.Hedberg ${ }^{25}$, M.Hennecke ${ }^{17}$, H.Herr ${ }^{8}$, J.Hoffman ${ }^{51}$, S-O.Holmgren ${ }^{43}$, P.J.Holt ${ }^{8}$, M.A.Houlden ${ }^{23}$, K.Hultqvist ${ }^{43}$, J.N.Jackson ${ }^{23}$, G.Jarlskog ${ }^{25}$, P.Jarry $^{39}$, D.Jeans ${ }^{34}$, E.K.Johansson ${ }^{43}$, P.D.Johansson ${ }^{43}$, P.Jonsson ${ }^{26}$, C.Joram ${ }^{8}$, L.Jungermann ${ }^{17}$, F.Kapusta ${ }^{24}$, S.Katsanevas ${ }^{26}$, E.Katsoufis ${ }^{31}$, G.Kernel ${ }^{42}$, B.P.Kersevan ${ }^{8,42}$, A.Kiiskinen ${ }^{15}$, B.T.King ${ }^{23}$, N.J.Kjaer ${ }^{8}$, P.Kluit ${ }^{30}$, P.Kokkinias ${ }^{11}$, C.Kourkoumelis ${ }^{3}$, O.Kouznetsov ${ }^{16}$, Z.Krumstein ${ }^{16}$, M.Kucharczyk ${ }^{18}$, J.Lamsa $^{1}$, G.Leder ${ }^{50}$, F.Ledroit ${ }^{14}$, L.Leinonen ${ }^{43}$, R.Leitner ${ }^{29}$, J.Lemonne ${ }^{2}$, V.Lepeltier ${ }^{20}$, T.Lesiak ${ }^{18}$, W.Liebig $^{52}$, D.Liko ${ }^{50}$, A.Lipniacka ${ }^{43}$, J.H.Lopes ${ }^{47}$, J.M.Lopez ${ }^{33}$, D.Loukas ${ }^{11}, \quad$ P.Lutz ${ }^{39}$, L.Lyons ${ }^{34}$, J.MacNaughton ${ }^{50}$, A.Malek ${ }^{52}$, S.Maltezos ${ }^{31}$, F.Mandl ${ }^{50}$, J.Marco ${ }^{40}$, R.Marco ${ }^{40}$, B.Marechal ${ }^{47}$, M.Margoni ${ }^{35}$, J-C.Marin $^{8}$, C.Mariotti ${ }^{8}$, A.Markou ${ }^{11}$, C.Martinez-Rivero ${ }^{40}$, J.Masik ${ }^{12}$, N.Mastroyiannopoulos ${ }^{11}$, F.Matorras ${ }^{40}$, C.Matteuzzi $^{28}$, F.Mazzucato ${ }^{35}$, M.Mazzucato ${ }^{35}$, R.Mc Nulty ${ }^{23}$, C.Meroni ${ }^{27}$, W.T.Meyer ${ }^{1}$, E.Migliore ${ }^{44}$,

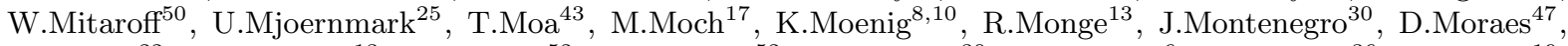
S.Moreno $^{22}$, P.Morettini ${ }^{13}$, U.Mueller ${ }^{52}$, K.Muenich ${ }^{52}$, M.Mulders ${ }^{30}$, L.Mundim ${ }^{6}$, W.Murray ${ }^{36}$, B.Muryn ${ }^{19}$, G.Myatt $^{34}$, T.Myklebust ${ }^{32}$, M.Nassiakou ${ }^{11}$, F.Navarria ${ }^{5}$, K.Nawrocki ${ }^{51}$, R.Nicolaidou ${ }^{39}$, M.Nikolenko ${ }^{16,9}$, A.Oblakowska-Mucha ${ }^{19}$, V.Obraztsov ${ }^{41}$, A.Olshevski ${ }^{16}$, A.Onofre ${ }^{22}$, R.Orava ${ }^{15}$, K.Osterberg ${ }^{15}$, A.Ouraou ${ }^{39}$, A.Oyanguren $^{49}$, M.Paganoni ${ }^{28}$, S.Paiano ${ }^{5}$, J.P.Palacios ${ }^{23}$, H.Palka ${ }^{18}$, Th.D.Papadopoulou ${ }^{31}$, L.Pape ${ }^{8}$, C.Parkes $^{23}$, F.Parodi ${ }^{13}$, U.Parzefall ${ }^{8}$, A.Passeri ${ }^{38}$, O.Passon ${ }^{52}$, L.Peralta ${ }^{22}$, V.Perepelitsa ${ }^{49}$, A.Perrotta ${ }^{5}$,

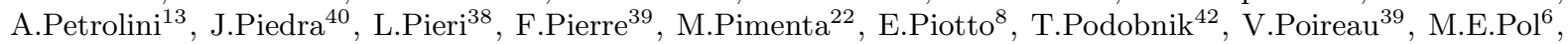
G.Polok $^{18}$, P.Poropat ${ }^{\dagger 46}$, V.Pozdniakov ${ }^{16}$, N.Pukhaeva ${ }^{2,16}$, A.Pullia ${ }^{28}$, J.Rames ${ }^{12}$, L.Ramler ${ }^{17}$, A.Read $^{32}$, P.Rebecchi ${ }^{8}$, J.Rehn ${ }^{17}$, D.Reid ${ }^{30}$, R.Reinhardt ${ }^{52}$, P.Renton ${ }^{34}$, F.Richard ${ }^{20}$, J.Ridky ${ }^{12}$, M.Rivero ${ }^{40}$, D.Rodriguez $^{40}$, A.Romero ${ }^{44}$, P.Ronchese ${ }^{35}$, E.Rosenberg ${ }^{1}$, P.Roudeau ${ }^{20}$, T.Rovelli ${ }^{5}$, V.Ruhlmann-Kleider ${ }^{39}$, D.Ryabtchikov ${ }^{41}$, A.Sadovsky ${ }^{16}$, L.Salmi ${ }^{15}$, J.Salt ${ }^{49}$, A.Savoy-Navarro ${ }^{24}$, U.Schwickerath ${ }^{8}$, A.Segar ${ }^{34}$, R.Sekulin ${ }^{36}$, M.Siebel ${ }^{52}$, A.Sisakian ${ }^{16}$, G.Smadja ${ }^{26}$, O.Smirnova ${ }^{25}$, A.Sokolov ${ }^{41}$, A.Sopczak ${ }^{21}$, R.Sosnowski ${ }^{51}$, T.Spassov ${ }^{8}$, M.Stanitzki ${ }^{17}$, A.Stocchi ${ }^{20}$, J.Strauss ${ }^{50}$, B.Stugu ${ }^{4}$, M.Szczekowski ${ }^{51}$, M.Szeptycka ${ }^{51}$, T.Szumlak ${ }^{19}$, T.Tabarelli $^{28}$, A.C.Taffard ${ }^{23}$, F.Tegenfeldt ${ }^{48}$, J.Timmermans ${ }^{30}$, L.Tkatchev ${ }^{16}$, M.Tobin ${ }^{23}$, S.Todorovova ${ }^{12}$,

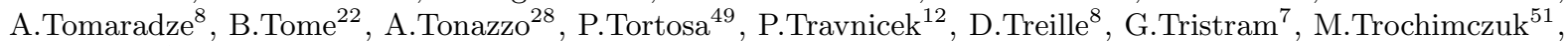
C.Troncon ${ }^{27}$, M-L.Turluer ${ }^{39}$, I.A.Tyapkin ${ }^{16}$, P.Tyapkin ${ }^{16}$, S.Tzamarias ${ }^{11}, \quad$ V.Uvarov ${ }^{41}$, G.Valenti ${ }^{5}$, P.Van Dam ${ }^{30}$, J.Van Eldik ${ }^{8}$, A.Van Lysebetten ${ }^{2}$, N.van Remortel ${ }^{2}$, I.Van Vulpen ${ }^{30}$, G.Vegni ${ }^{27}$, F.Veloso ${ }^{22}$, W.Venus $^{36}$, F.Verbeure ${ }^{2}$, P.Verdier ${ }^{26}$, V.Verzi ${ }^{37}$, D.Vilanova ${ }^{39}$, L.Vitale ${ }^{46}$, V.Vrba ${ }^{12}$, H.Wahlen ${ }^{52}$, 
A.J.Washbrook ${ }^{23}$, C.Weiser ${ }^{17}$, D.Wicke ${ }^{8}$, J.Wickens ${ }^{2}$, G.Wilkinson ${ }^{34}$, M.Winter ${ }^{9}$, M.Witek ${ }^{18}$, O.Yushchenko ${ }^{41}$, A.Zalewska $^{18}$, P.Zalewski ${ }^{51}$, D.Zavrtanik ${ }^{42}$, N.I.Zimin ${ }^{16}$, A.Zintchenko ${ }^{16}$, M.Zupan ${ }^{11}$

\footnotetext{
${ }^{1}$ Department of Physics and Astronomy, Iowa State University, Ames IA 50011-3160, USA

${ }^{2}$ Physics Department, Universiteit Antwerpen, Universiteitsplein 1, B-2610 Antwerpen, Belgium and IIHE, ULB-VUB, Pleinlaan 2, B-1050 Brussels, Belgium

and Faculté des Sciences, Univ. de l'Etat Mons, Av. Maistriau 19, B-7000 Mons, Belgium

${ }^{3}$ Physics Laboratory, University of Athens, Solonos Str. 104, GR-10680 Athens, Greece

${ }^{4}$ Department of Physics, University of Bergen, Allégaten 55, NO-5007 Bergen, Norway

${ }^{5}$ Dipartimento di Fisica, Università di Bologna and INFN, Via Irnerio 46, IT-40126 Bologna, Italy

${ }^{6}$ Centro Brasileiro de Pesquisas Físicas, rua Xavier Sigaud 150, BR-22290 Rio de Janeiro, Brazil and Depto. de Física, Pont. Univ. Católica, C.P. 38071 BR-22453 Rio de Janeiro, Brazil and Inst. de Física, Univ. Estadual do Rio de Janeiro, rua São Francisco Xavier 524, Rio de Janeiro, Brazil

${ }^{7}$ Collège de France, Lab. de Physique Corpusculaire, IN2P3-CNRS, FR-75231 Paris Cedex 05, France

${ }^{8} \mathrm{CERN}, \mathrm{CH}-1211$ Geneva 23, Switzerland

${ }^{9}$ Institut de Recherches Subatomiques, IN2P3 - CNRS/ULP - BP20, FR-67037 Strasbourg Cedex, France

${ }^{10}$ Now at DESY-Zeuthen, Platanenallee 6, D-15735 Zeuthen, Germany

${ }^{11}$ Institute of Nuclear Physics, N.C.S.R. Demokritos, P.O. Box 60228, GR-15310 Athens, Greece

${ }^{12}$ FZU, Inst. of Phys. of the C.A.S. High Energy Physics Division, Na Slovance 2, CZ-180 40, Praha 8, Czech Republic

${ }^{13}$ Dipartimento di Fisica, Università di Genova and INFN, Via Dodecaneso 33, IT-16146 Genova, Italy

${ }^{14}$ Institut des Sciences Nucléaires, IN2P3-CNRS, Université de Grenoble 1, FR-38026 Grenoble Cedex, France

${ }^{15}$ Helsinki Institute of Physics, HIP, P.O. Box 9, FI-00014 Helsinki, Finland

${ }^{16}$ Joint Institute for Nuclear Research, Dubna, Head Post Office, P.O. Box 79, RU-101 000 Moscow, Russian Federation

${ }^{17}$ Institut für Experimentelle Kernphysik, Universität Karlsruhe, Postfach 6980, DE-76128 Karlsruhe, Germany

${ }^{18}$ Institute of Nuclear Physics,Ul. Kawiory 26a, PL-30055 Krakow, Poland

${ }^{19}$ Faculty of Physics and Nuclear Techniques, University of Mining and Metallurgy, PL-30055 Krakow, Poland

${ }^{20}$ Université de Paris-Sud, Lab. de l'Accélérateur Linéaire, IN2P3-CNRS, Bât. 200, FR-91405 Orsay Cedex, France

${ }^{21}$ School of Physics and Chemistry, University of Lancaster, Lancaster LA1 4YB, UK

${ }^{22}$ LIP, IST, FCUL - Av. Elias Garcia, 14-1 ${ }^{\circ}$, PT-1000 Lisboa Codex, Portugal

${ }^{23}$ Department of Physics, University of Liverpool, P.O. Box 147, Liverpool L69 3BX, UK

${ }^{24}$ LPNHE, IN2P3-CNRS, Univ. Paris VI et VII, Tour 33 (RdC), 4 place Jussieu, FR-75252 Paris Cedex 05, France

${ }^{25}$ Department of Physics, University of Lund, Sölvegatan 14, SE-223 63 Lund, Sweden

${ }^{26}$ Université Claude Bernard de Lyon, IPNL, IN2P3-CNRS, FR-69622 Villeurbanne Cedex, France

${ }^{27}$ Dipartimento di Fisica, Università di Milano and INFN-MILANO, Via Celoria 16, IT-20133 Milan, Italy

${ }^{28}$ Dipartimento di Fisica, Univ. di Milano-Bicocca and INFN-MILANO, Piazza della Scienza 2, IT-20126 Milan, Italy

${ }^{29}$ IPNP of MFF, Charles Univ., Areal MFF, V Holesovickach 2, CZ-180 00, Praha 8, Czech Republic

${ }^{30}$ NIKHEF, Postbus 41882, NL-1009 DB Amsterdam, The Netherlands

${ }^{31}$ National Technical University, Physics Department, Zografou Campus, GR-15773 Athens, Greece

${ }^{32}$ Physics Department, University of Oslo, Blindern, NO-0316 Oslo, Norway

${ }^{33}$ Dpto. Fisica, Univ. Oviedo, Avda. Calvo Sotelo s/n, ES-33007 Oviedo, Spain

${ }^{34}$ Department of Physics, University of Oxford, Keble Road, Oxford OX1 3RH, UK

${ }^{35}$ Dipartimento di Fisica, Università di Padova and INFN, Via Marzolo 8, IT-35131 Padua, Italy

${ }^{36}$ Rutherford Appleton Laboratory, Chilton, Didcot OX11 OQX, UK

${ }^{37}$ Dipartimento di Fisica, Università di Roma II and INFN, Tor Vergata, IT-00173 Rome, Italy

${ }^{38}$ Dipartimento di Fisica, Università di Roma III and INFN, Via della Vasca Navale 84, IT-00146 Rome, Italy

${ }^{39}$ DAPNIA/Service de Physique des Particules, CEA-Saclay, FR-91191 Gif-sur-Yvette Cedex, France

${ }^{40}$ Instituto de Fisica de Cantabria (CSIC-UC), Avda. los Castros s/n, ES-39006 Santander, Spain

${ }^{41}$ Inst. for High Energy Physics, Serpukov P.O. Box 35, Protvino, (Moscow Region), Russian Federation

42 J. Stefan Institute, Jamova 39, SI-1000 Ljubljana, Slovenia and Laboratory for Astroparticle Physics,

Nova Gorica Polytechnic, Kostanjeviska 16a, SI-5000 Nova Gorica, Slovenia,

and Department of Physics, University of Ljubljana, SI-1000 Ljubljana, Slovenia

${ }^{43}$ Fysikum, Stockholm University, Box 6730, SE-113 85 Stockholm, Sweden

${ }^{44}$ Dipartimento di Fisica Sperimentale, Università di Torino and INFN, Via P. Giuria 1, IT-10125 Turin, Italy

${ }^{45}$ INFN,Sezione di Torino, and Dipartimento di Fisica Teorica, Università di Torino, Via P. Giuria 1, IT-10125 Turin, Italy

${ }^{46}$ Dipartimento di Fisica, Università di Trieste and INFN, Via A. Valerio 2, IT-34127 Trieste, Italy and Istituto di Fisica, Università di Udine, IT-33100 Udine, Italy

${ }^{47}$ Univ. Federal do Rio de Janeiro, C.P. 68528 Cidade Univ., Ilha do Fundão BR-21945-970 Rio de Janeiro, Brazil

${ }^{48}$ Department of Radiation Sciences, University of Uppsala, P.O. Box 535, SE-751 21 Uppsala, Sweden

${ }^{49}$ IFIC, Valencia-CSIC, and D.F.A.M.N., U. de Valencia, Avda. Dr. Moliner 50, ES-46100 Burjassot (Valencia), Spain

${ }^{50}$ Institut für Hochenergiephysik, Österr. Akad. d. Wissensch., Nikolsdorfergasse 18, AT-1050 Vienna, Austria

${ }^{51}$ Inst. Nuclear Studies and University of Warsaw, Ul. Hoza 69, PL-00681 Warsaw, Poland

${ }^{52}$ Fachbereich Physik, University of Wuppertal, Postfach 100 127, DE-42097 Wuppertal, Germany

$\dagger$ deceased
} 


\section{Introduction}

In the Standard Model, $\mathrm{B}_{q}^{0}-\overline{\mathrm{B}_{q}^{0}}(q=d, s)$ mixing is a direct consequence of second order weak interactions. Starting with a $\mathrm{B}_{q}^{0}$ meson produced at time $t=0$, the probability density $\mathcal{P}$ to observe a $\mathrm{B}_{q}^{0}$ decaying at the proper time $t$ can be written, neglecting effects from $\mathrm{CP}$ violation:

$$
\mathcal{P}\left(\mathrm{B}_{q}^{0} \rightarrow \mathrm{B}_{q}^{0}\right)=\frac{\Gamma_{q}}{2} e^{-\Gamma_{q} t}\left[\cosh \left(\frac{\Delta \Gamma_{q}}{2} t\right)+\cos \left(\Delta m_{q} t\right)\right] .
$$

Here $\Gamma_{q}=\frac{\Gamma_{q}^{H}+\Gamma_{q}^{L}}{2}, \Delta \Gamma_{q}=\Gamma_{q}^{H}-\Gamma_{q}^{L}$, and $\Delta m_{q}=m_{q}^{H}-m_{q}^{L}$, where $H$ and $L$ denote respectively the heavy and light physical states. The oscillation period gives a direct measurement of the mass difference between the two physical states. The Standard Model predicts that $\Delta \Gamma \ll \Delta m[1]$. Neglecting a possible difference between the lifetimes of the heavy and light mass eigenstates, the above expression simplifies to:

$$
\mathcal{P}_{\mathrm{B}_{q}^{0}}^{\text {unmix }}=\mathcal{P}\left(\mathrm{B}_{q}^{0} \rightarrow \mathrm{B}_{q}^{0}\right)=\frac{1}{2 \tau_{q}} e^{-\frac{t}{\tau_{q}}}\left[1+\cos \left(\Delta m_{q} t\right)\right]
$$

and similarly:

$$
\mathcal{P}_{\mathrm{B}_{q}^{0}}^{\text {mix. }}=\mathcal{P}\left(\mathrm{B}_{q}^{0} \rightarrow \overline{\mathrm{B}_{q}^{0}}\right)=\frac{1}{2 \tau_{q}} e^{-\frac{t}{\tau_{q}}}\left[1-\cos \left(\Delta m_{q} t\right)\right]
$$

where $\tau_{q}$ is the lifetime of the $\mathrm{B}_{q}^{0}$.

In the Standard Model, the $\mathrm{B}_{q}^{0}-\overline{\mathrm{B}_{q}^{0}}(q=\mathrm{d}, \mathrm{s})$ mass difference $\Delta m_{q}$ (having kept only the dominant top quark contribution) can be expressed as follows [1]:

$$
\Delta m_{q}=\frac{G_{F}^{2}}{6 \pi^{2}}\left|V_{t b}\right|^{2}\left|V_{t q}\right|^{2} m_{t}^{2} m_{\mathrm{B}_{\mathrm{q}}} f_{\mathrm{B}_{\mathrm{q}}}^{2} B_{\mathrm{B}_{\mathrm{q}}} \eta_{B} F\left(\frac{m_{t}^{2}}{m_{W}^{2}}\right) .
$$

In this expression $G_{F}$ is the Fermi coupling constant; $F\left(x_{t}\right)$, with $x_{t}=\frac{m_{t}^{2}}{m_{W}^{2}}$, results from the evaluation of the box diagram and has a smooth dependence on $x_{t}$. $\eta_{B}$ is a QCD correction factor obtained at next-to-leading order in perturbative QCD. The dominant uncertainties in Eq.(3) come from the evaluation of the B meson decay constant $f_{\mathrm{B}_{\mathrm{q}}}$ and of the "bag" parameter $B_{\mathrm{B}_{\mathrm{q}}}[2]$. In terms of the Wolfenstein parametrization [3], the two elements of the $V_{C K M}$ matrix are equal to:

$$
\left|V_{t d}\right|=A \lambda^{3} \sqrt{(1-\rho)^{2}+\eta^{2}} \quad ; \quad\left|V_{t s}\right|=A \lambda^{2},
$$

neglecting terms of order $O\left(\lambda^{4}\right)$. At this order $\left|V_{t s}\right|$ is independent of $\rho$ and $\eta$ and is equal to $\left|V_{c b}\right|$. Eq. (3) relates $\Delta m_{d}$ to $\left|V_{t d}\right|$. It defines a circle in the $\rho-\eta$ plane. Nevertheless the precision on $\Delta m_{d}$ cannot be fully exploited due to the large uncertainty which originates in the evaluation of the non-perturbative QCD parameters.

The ratio between the Standard Model expectations for $\Delta m_{d}$ and $\Delta m_{s}$ is given by:

$$
\frac{\Delta m_{d}}{\Delta m_{s}}=\frac{m_{\mathrm{B}_{\mathrm{d}}} f_{\mathrm{B}_{\mathrm{d}}}^{2} B_{\mathrm{B}_{\mathrm{d}}} \eta_{\mathrm{B}_{\mathrm{d}}}}{m_{\mathrm{B}_{\mathrm{s}}} f_{\mathrm{B}_{\mathrm{s}}}^{2} B_{\mathrm{B}_{\mathrm{s}}} \eta_{\mathrm{B}_{\mathrm{s}}}} \frac{\left|V_{t d}\right|^{2}}{\left|V_{t s}\right|^{2}} .
$$

A measurement or a limit on the ratio $\frac{\Delta m_{d}}{\Delta m_{s}}$ gives a circular constraint in the $\rho-\eta$ plane. This ratio depends only on the ratio of the non-perturbative QCD parameters which is expected to be better determined than their absolute values which occur in Eq. (3). Using constraints on $\rho$ and $\eta$ from existing measurements (except those on $\Delta m_{s}$ ), the 
distribution for the expected values of $\Delta m_{s}$ can be obtained. It has been shown that $\Delta m_{s}$ should lie, at $95 \%$ C.L., between 9.7 and $23.2 \mathrm{ps}^{-1}[2]$.

Using the DELPHI data, several analyses searching for $\mathrm{B}_{s}^{0}-\overline{\mathrm{B}_{s}^{0}}$ oscillations have been performed on selected event samples of exclusively reconstructed $\mathrm{B}_{s}^{0}$ mesons, $\mathrm{D}_{s}$-lepton pairs, $\mathrm{D}_{s}$-hadron pairs and events with a high transverse momentum lepton [4]. In this analysis events with a high transverse momentum lepton have been removed and the remaining events are used to search for $\mathrm{B}_{s}^{0}$ oscillations and to measure the $\mathrm{B}_{d}^{0}$ oscillation frequency. Two analyses will be described: one inclusive vertex analysis based on a probabilistic approach using the data set from 1992 to 2000 and one based on neural networks optimized for high values of $\Delta m_{s}$ using only the 1994 data. To avoid overlap with other analyses [4], events with a high transverse momentum lepton are removed from the sample. Both analyses reconstruct an inclusive secondary vertex which is used to estimate the proper time. Events that mix are selected using a tag based on several separating variables which are combined using probabilities or neural networks respectively. The neural network analysis will provide a check and a confirmation of the results and in particular of the sensitivity at high values of $\Delta m_{s}$.

The inclusive vertex analysis is presented in section 2 , describing the secondary vertex and proper time reconstruction, the production and decay tags and the fitting programme. The measurement of the $\mathrm{B}_{d}^{0}-\overline{\mathrm{B}_{d}^{0}}$ oscillation frequency is described in section 2.7 and the results of the search for $\mathrm{B}_{s}^{0}-\overline{\mathrm{B}_{s}^{0}}$ oscillations are presented in section 2.8 . In section 3 , the neural network analysis is described, while the conclusions are presented in section 4 .

The results presented in this paper will be combined later with other DELPHI and LEP results.

\section{Inclusive vertex analysis}

For a description of the DELPHI detector and of its performance the reader is referred to [7]. The analysis described in this paper used the precise tracking based on the silicon microvertex detector to reconstruct the primary and secondary vertex. To estimate the $\mathrm{B}$ momentum and direction, the neutral particles detected in the electromagnetic and hadronic calorimeter and the reconstructed tracks were used. Muon identification was based on the hits in the muon chambers being associated with a track. Electrons were identified using tracks associated with a shower in the electromagnetic calorimeter. The $\mathrm{dE} / \mathrm{dx}$ energy loss measurement in the Time Projection Chamber and the Cherenkov light detected in the RICH were used to separate pions (and also electrons or muons) from kaons and protons.

Tracks were selected if they satisfied the following criteria: their particle momentum was above $200 \mathrm{MeV} / \mathrm{c}$, their tracklength was at least $30 \mathrm{~cm}$, their relative momentum error was less than $130 \%$, their polar angle was between $20^{\circ}$ and $160^{\circ}$ and their impact parameter with respect to the primary vertex was less than $4 \mathrm{~cm}$ in the $x y$ plane (perpendicular to the beam) and $10 \mathrm{~cm}$ in $z$ (along the beam direction). Neutral particles had to deposit at least $500 \mathrm{MeV}$ in the calorimeters and their polar angle had to lie between $2^{\circ}$ and $178^{\circ}$.

To select hadronic events it was required that more than 7 tracks of charged particles were accepted with a total energy above $15 \mathrm{GeV}$. The thrust direction was determined using charged and neutral particles and its polar angle was required to satisfy $\left|\cos \left(\theta_{\text {thrust }}\right)\right|<0.8$. The event was divided into hemispheres by a plane perpendicular to the thrust axis. In each hemisphere the total energy from charged and neutral particles 
had to be larger than $5 \mathrm{GeV}$. In total about 4 million hadronic $\mathrm{Z}$ decays were selected from which 3.5 million were taken in the LEP I phase (1992-1995) and 500k were collected as calibration data in the LEP II phase (1996-2000).

Using tracks with vertex detector information, the primary vertex was fitted using the average beamspot as a constraint [5]. For each track the impact parameter with respect to the primary vertex was calculated and the lifetime sign determined as explained in the paper quoted above. The $\mathrm{b}$ tagging probability ${ }^{1} P_{E}^{+}$is a measure of the consistency of these track impact parameters with the hypothesis that all selected tracks came from the event's production vertex. Events without long-lived particles should have a uniform distribution of $P_{E}^{+}$, while those containing a b-quark tend to have small values. In the 1992 and 1993 data the vertex detector measured only the $R \phi\left(\mathrm{R}\right.$ being defined as $\sqrt{x^{2}+y^{2}}$ and $\phi$ the azimuthal angle) coordinate, while from 1994 to 2000 the $z$ coordinate was also measured. In the 1992-1993 data, events were selected if the b tagging variable $P_{E}^{+}$was less than 0.1, whereas in the 1994-2000 data, the cut could be placed at 0.015.

Jets were reconstructed using charged and neutral particles by the LUCLUS [6] jet algorithm with an invariant mass cut DJOIN of $6 \mathrm{GeV} / \mathrm{c}^{2}$. Leptons were identified and their transverse momentum with respect to the jet axis was determined. Loosely identified muons with momenta above $3 \mathrm{GeV} / \mathrm{c}$ were accepted as well as standard and tightly identified muon with momenta above $2 \mathrm{GeV} / \mathrm{c}$. The reader is referred to [7] for the identification criteria. Events with a standard or tightly identified muons with momentum above $3 \mathrm{GeV} / \mathrm{c}$ and a transverse momentum above $1.2 \mathrm{GeV} / \mathrm{c}$ were removed from the selected event sample. This was done to avoid overlap with other analyses that use leptons [4] with a high transverse momentum. For electron identification a neural network was used with a cut value that corresponds to $75 \%$ efficiency [7]. The electron had to have a momentum above $2 \mathrm{GeV} / \mathrm{c}$. Electrons with a momentum below $3 \mathrm{GeV} / \mathrm{c}$ had to pass a cut value that corresponds to $65 \%$ efficiency. Again to avoid overlap with other analyses that use high transverse momentum leptons, events with an electron with momentum above $3 \mathrm{GeV} / \mathrm{c}$ and a transverse momentum above $1.2 \mathrm{GeV} / \mathrm{c}$ satisfying a cut value that corresponds to $65 \%$ efficiency were removed. The selected electrons and muons will henceforth be referred to as soft leptons.

Samples of hadronic $\mathrm{Z}$ decays (4 million events) and of $\mathrm{Z}$ bosons decaying only into $\mathrm{b} \overline{\mathrm{b}}$ quark pairs (2 million events) were simulated using the Monte Carlo generator programme JETSET7.3 [6] with DELPHI tuned JETSET parameters and updated b and c decay tables [8]. The detailed response of the DELPHI detector was simulated [9].

\subsection{Secondary vertex reconstruction}

The secondary vertex reconstruction and proper time determination procedures are identical for events with or without a soft lepton. First the probability $P_{i}$ that a charged or a neutral particle comes from the secondary (bottom or charm) vertex was parametrized ${ }^{2}$. The following information was used for tracks: the lifetime-signed impact parameters and their errors (in $R \phi$ and $R z$ ), the transverse momentum with respect to the jet axis, the muon and electron identification and the rapidity ${ }^{3}$ with respect to the jet axis. For neutral particles the transverse momentum and rapidity were used. For each of these quantities the probability was parametrized using the simulation. The total probability was obtained by combining these individual probabilities assuming they are independent.

${ }^{1} E$ refers to the fact that the total event was used and the + sign means that the lifetime sign had to be positive.

${ }^{2}$ Thus a $P_{i}$ value of 0.8 means that 80 percent of the selected particles will come from the secondary vertex.

${ }^{3}$ For calculating rapidities, charged and neutral particles were assigned the pion mass. 
To start the first level secondary vertex fit, tracks were selected with at least one associated hit in the vertex detector and a probability $P_{i}$ larger than $60 \%$. The decay length - i.e. the 3-D decay distance - per track was determined by calculating the point of closest approach of the track to the B particle trajectory which was approximated by a track coming from the primary vertex and having the direction of the reconstructed jet. The first level secondary vertex was fitted using the measured decay lengths per track and their errors, the azimuthal and polar angles of the tracks and the B trajectory. The result of this approximate fit was a decay length, its error and a $\chi^{2}$ of the fit. Further, the $\chi_{t}^{2}$ contribution of each track to the total $\chi^{2}$ was determined. To remove tracks coming from the primary vertex the following iterative procedure was performed: if the secondary vertex was reconstructed with more than two tracks, the track upstream of the vertex (i.e. closer to the fitted primary vertex) with the largest $\chi_{t}^{2}$ was removed if its $\chi_{t}^{2}$ was larger than 4. Secondly, tracks were removed that did not combine with any of the other tracks. To achieve this, all two track combinations were made and the number of good matches was counted. A good match was defined as a two track vertex that was within 2 standard deviations of the fitted secondary vertex. For each track, the fraction $f_{\text {good }}$ of good matches to the total number of combinations was determined. The track with the smallest $f_{\text {good }}$ value was removed if its value was below $20 \%$, and then the first level vertex fit was redone. The procedure ends when no track could be removed by the listed criteria.

At the end of this procedure a full vertex fit was performed using the measured track parameters and the corresponding covariance matrices. To the list of tracks selected for the fit, the B-track with its covariance matrix was added as a constraint. For each track the impact parameter and its error with respect to the fitted secondary vertex were calculated. The global $\chi^{2}$ of the fit was defined as the sum of the squares of the track impact parameters divided by corresponding uncertainties (in $R \phi$ and $R z$ ). As a result the $\mathrm{B}$ decay length and its error were obtained.

The presence of tracks from charm particle decays in the vertex fit has two effects. Firstly, the fitted vertex does not coincide with the B vertex, but is some average between the $\mathrm{B}$ and $\mathrm{D}$ vertex positions. Secondly, the $\chi^{2}$ of the vertex increases because of the charm decay length. It was therefore important to remove as much as possible the decay products of charmed particles from the vertex fit. For this purpose the probability that a track came from charm was evaluated on the basis of kinematic and vertex information. For example, the momentum distribution of particles from charm, in the B rest frame, is softer than that for particles from B decays. Secondly, a particle from charm decay is produced downstream of the fitted vertex, while a particle from a B hadron originated upstream of this vertex. Two new vertex fits were performed. In the first, one particle that most likely originates from charm was removed. In the second fit, the two particles most likely to come from charm were removed.

Using the simulation, an estimate was made of the B decay length and of its error, using as an input the fitted decay length, its associated (or raw) error, the $\chi^{2}$ and the number of fitted tracks. The expected error on the B decay length was parametrized in the same way. This was done for the three vertex fits (removing 0,1 and 2 particles as described in the previous paragraph). Removing 1 or 2 particles has the advantage of reducing the bias caused by the presence of particles from charm. On the other hand the resolution is degraded if a track is removed. Due to the fact that the $\chi^{2}$ is sensitive to the presence of particles from charm, part of the bias is corrected for in the parametrization of the B decay length. Finally, out of the three vertex fit results, the result with the 
smallest expected error on the B decay length was chosen. In $51 \%$ of the cases no track was removed, in $36 \%$ one track and in $13 \%$ two tracks were removed.

In Figures $1 \mathrm{a}$ and $\mathrm{b}$ the raw error as it comes out of the full vertex fit and the reconstructed minus the $\mathrm{B}$ decay distance divided by the raw error are shown for the 1994-1995 simulated events. The tail due to the presence of charmed particles can be clearly observed. Figures $1 \mathrm{c}$ and d show the expected error and the reconstructed minus the simulated B decay distance after applying the correction procedure described above. The latter distribution is clearly more Gaussian and its width is close to unity.
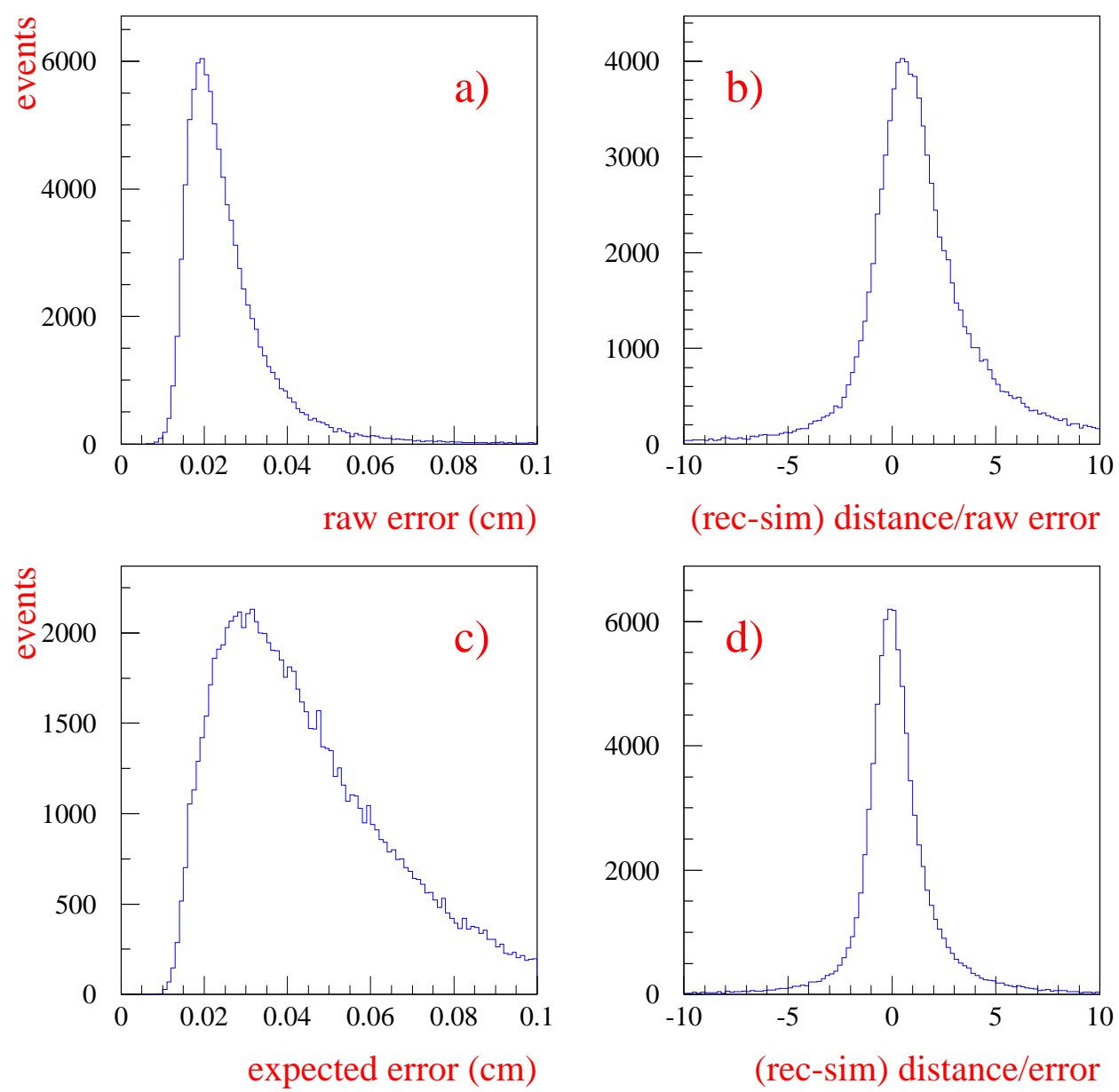

Figure 1: Figure a) shows the expected or raw error, Figure b) the reconstructed minus the simulated B decay distance divided by the raw error for the 1994-1995 simulation. Figures c) and d) show the expected error and the reconstructed minus the simulated B decay distance after applying the procedure described in the text.

\subsection{Proper time reconstruction}

To determine the proper time, the momentum of the $\mathrm{B}$ hadron had to be measured. An estimate of the energy of the $\mathrm{b}$ jet was made, applying energy-momentum conservation to the whole event. The masses of the jet containing the B hadron and of the system 
formed by the remaining charged and neutral particles, labelled respectively $M_{1}$ and $M_{2}$, were measured. The b jet energy was obtained as:

$$
E_{j e t}=E_{c m s} / 2-\left(M_{2}^{2}-M_{1}^{2}\right) /\left(2 E_{c m s}\right),
$$

where $E_{c m s}$ is the centre of mass energy. This significantly improved the b jet energy resolution. The B energy was determined as:

$$
E_{B}=\frac{\sum_{i} P_{i} E_{i}}{\sum_{i} E_{i}} E_{j e t}
$$

where $E_{i}$ is the energy of the charged or neutral particle and $P_{i}$ is the probability that a particle comes from the decay of a $\mathrm{B}$ hadron (see section 2.1).

The momentum of the B hadron was determined from the B energy and a small correction typically of order $10 \%$ was applied as a function of the following quantities: the weighted (with $P_{i}$ ) number of charged and neutral particles, the ratio of the raw $\mathrm{B}$ energy $\left(\sum_{i} P_{i} E_{i}\right)$ to the jet energy $E_{j e t}$, the invariant mass $M_{1}$, the ratio of the charged over the total raw $\mathrm{B}$ energy and the number of jets. The corrections were obtained from the simulation. The reconstructed B momentum is shown in Figure 2.

The expected error was parametrized as a function of the uncorrected B energy and of the jet energy. It lies between 3 and $9 \mathrm{GeV} / \mathrm{c}$ and is on the average equal to $5 \mathrm{GeV} / \mathrm{c}$. The reconstructed minus simulated $\mathrm{B}$ momentum divided by the expected error for simulated events is shown in Figure 2.

The proper time $t$ was calculated using:

$$
t=\frac{m L}{p}
$$

where $m$ is the $\mathrm{B}$ mass, $L$ the decay length and $p$ the estimated $\mathrm{B}$ momentum. The expected error $\sigma_{t}$ on the proper time was estimated using:

$$
\sigma_{t}=\sqrt{\left(\frac{m \delta L}{p}\right)^{2}+\left(\frac{m L \delta p}{p^{2}}\right)^{2}},
$$

where $\delta L$ is the expected error on the decay length and $\delta p$ is the error on the momentum. The data were divided into eight categories according to the value of the proper time resolution. This division was made because most of the sensitivity at high values of $\Delta m_{s}$ came from events with the best proper time resolution. The cuts are given in Table 1. To fall into the first category, the expected resolution had to be smaller than $0.12+0.07 t$ ps ( $t$ in ps units). Events with a resolution worse than $0.35+0.2 t$ ps were rejected.

The first four categories refer to events with a soft lepton and the last four to events with only an inclusive vertex. The soft lepton sample consists of 155023 events. The latter sample will be referred to as the inclusive vertex sample and consists of 614577 events. The proper time resolutions for the last four classes are shown in Figure 3.

The systematic error on the decay length resolution was estimated to be $\pm 10 \%$. This number was obtained in the following way. First, a comparison of data and simulation for the expected decay length error (see Fig. 4) showed that the data show a discrepancy for a scale error of less than $\pm 5 \%$. Secondly, the description of the impact parameters of the tracks with negative lifetime sign allow for a scaling of the associated error of less than $\pm 5 \%$ [5]. Finally, a study was made of the amplitude error (see section 2.8) as a function of $\Delta m_{s}$ comparing data and simulation. The amplitude error increases because of the finite proper time resolution. The amplitude error for data and simulation are in 
1992-2000 data
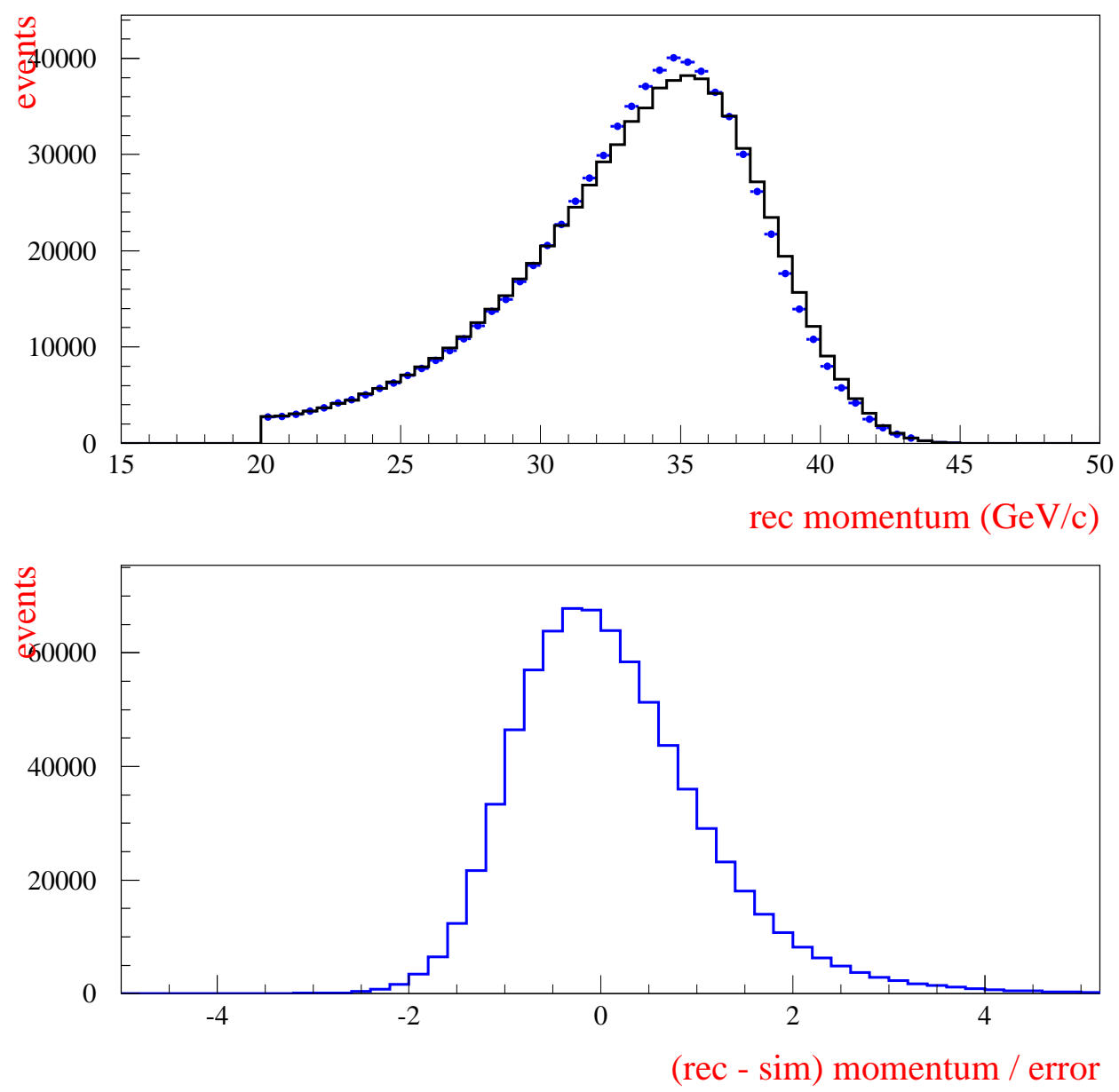

Figure 2: Upper diagram: Reconstructed B momentum. The dots correspond to the 1992 to 2000 data, the solid line to the measured momentum distribution obtained from simulated events. Lower diagram: The reconstructed minus the true B momentum divided by the expected error for simulated events.

agreement within $\pm 10 \%$. This is mainly due to the fact that the numbers of events in each category agree for data and simulation.

The systematic error on the momentum resolution was estimated to be $\pm 10 \%$. This number was obtained in the following way. Comparing the observed momentum in a hemisphere with the expected momentum in that hemisphere - obtained using energy and momentum conservation - for data and simulation, it was found that the momentum resolution agreed to better than $\pm 10 \%$. Finally, the study of the amplitude error, mentioned above, showed that the amplitude error for data and simulation was in agreement within $\pm 10 \%$. 


\begin{tabular}{|c|c|c|c|c|}
\hline category & 1 & 2 & 3 & 4 \\
\hline$\sigma_{t}(p s)$ & $0.12+0.07 t$ & $0.18+0.08 t$ & $0.25+0.1 t$ & $0.35+0.2 t$ \\
soft lepton sample & $22740(5533)$ & $41597(10598)$ & $42835(12091)$ & $47851(15620)$ \\
\hline category & 5 & 6 & 7 & 8 \\
\hline$\sigma_{t}(p s)$ & $0.12+0.07 t$ & $0.18+0.08 t$ & $0.25+0.1 t$ & $0.35+0.2 t$ \\
inclusive vertex sample & $68875(16476)$ & $146075(36633)$ & $171859(47702)$ & $227768(73809)$ \\
\hline
\end{tabular}

Table 1: Cuts on the resolution $\sigma_{t}$ and total number of selected events (in parenthesis the number of events corresponding to the 92-93 data) for the different categories.

\section{Inclusive vertices}
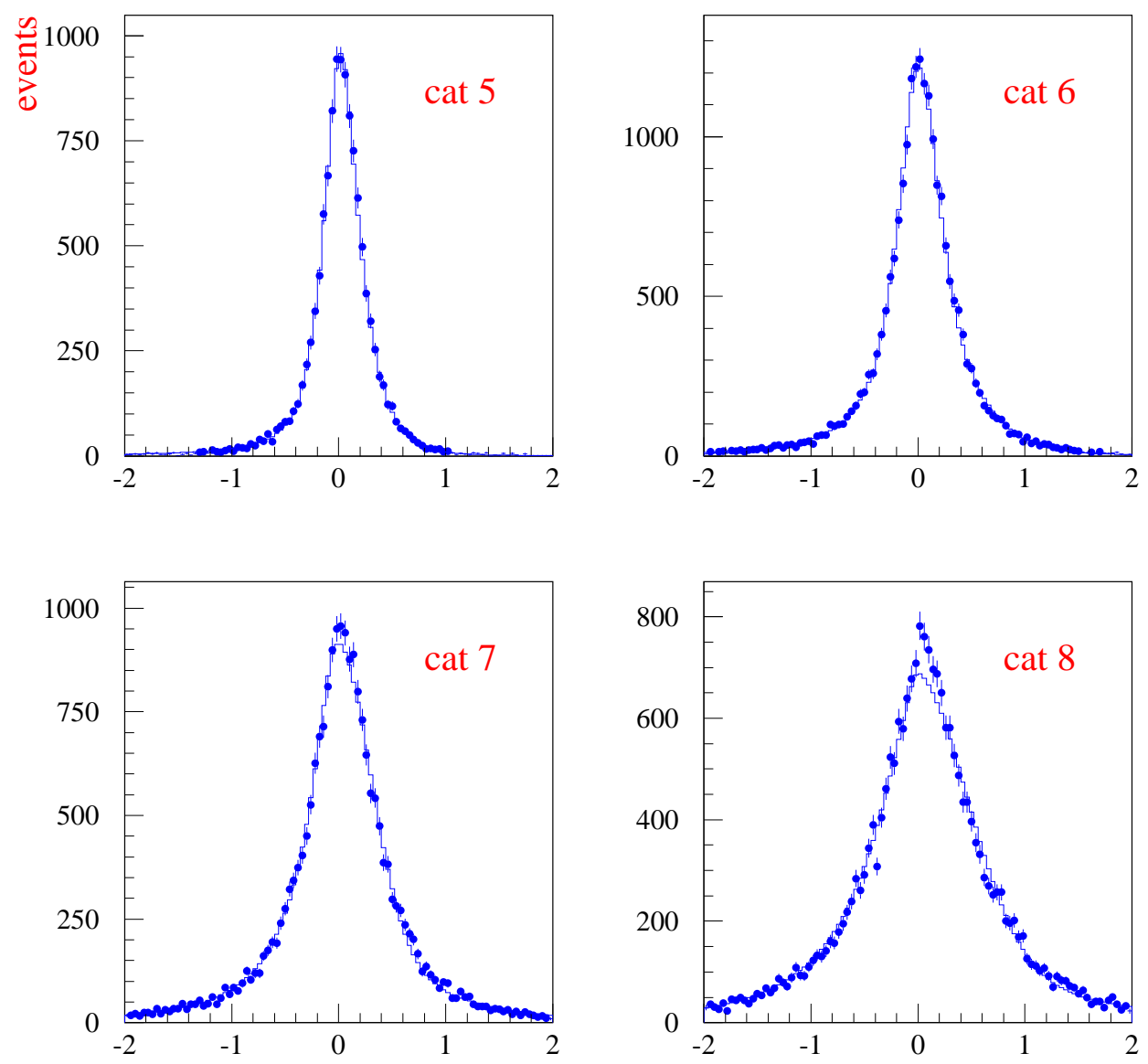

reconstructed-generated time $(\mathrm{ps})$

Figure 3: Reconstructed minus generated proper time for the inclusive B vertex sample corresponding to categories 5 to 8 . The dots correspond to the simulated data and the histograms to the parametrization of the resolution function (see section 2.5). 


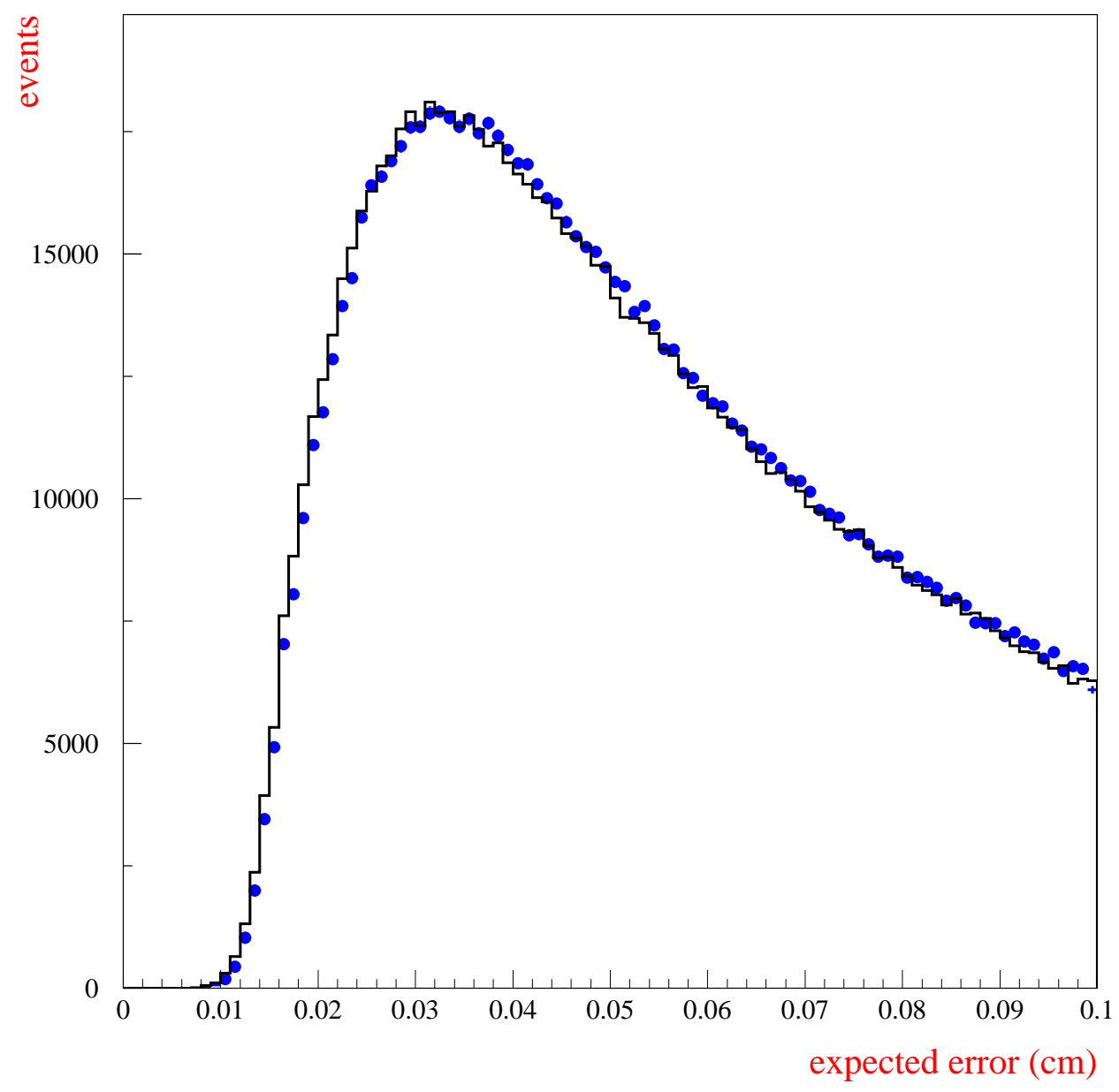

Figure 4: The expected error on the decay length for 1992-2000 data (points with error bars) and simulation (solid line). 


\subsection{Production and decay tag}

To distinguish between events in which the neutral B meson has mixed or not, a production and a decay tag were defined. They give, respectively, the b-flavor content (ie $\mathrm{b}$ or $\overline{\mathrm{b}}$ ) of the $\mathrm{B}$ hadron at production and decay times. In this analysis both the production and decay tags were optimized for $\mathrm{B}_{s}^{0}$ mesons. In $\mathrm{Z}$ decays, $\mathrm{b}$ and $\overline{\mathrm{b}}$ quarks are produced back to back, in pairs. The hemisphere opposite to the decaying B can therefore be used to tag the flavour at production time. This will be called the opposite side production tag which is obtained from a combination of several variables:

- the average charge of a sample of tracks, attached to the b-jet, and enriched in b-decay products:

$$
Q_{\text {jet }}=\sum q_{i} p_{i L} / \sum p_{i L} \text { with } P_{i}>0.5
$$

where $p_{i L}$ is the component of the momentum of the particle along the jet axis direction, and $P_{i}$ is its probability that it is a $\mathrm{B}$ decay product, as defined at the beginning of section 2.1

- the average charge of a sample of tracks, attached to the b-jet, and enriched in b-fragmentation products:

$$
Q_{f}=\sum q_{i} p_{i L}\left(P_{i}<0.5\right) / \sum p_{i L}\left(\text { all } P_{i}\right) .
$$

Note that the denominator sums over all particles, because the fraction of the total longitudinal momentum that is coming from fragmentation particles is relevant;

- the charge and momentum $p^{\star}$ of any identified lepton, in the B rest frame, which is determined from the inclusively reconstructed $\mathrm{B}$ momentum vector;

- the heavy particle charge for an identified kaon or proton and its momentum $p^{\star}$ in the $\mathrm{B}$ rest frame.

Using simulation, distributions for these variables were obtained for $\mathrm{B}$ and $\overline{\mathrm{B}}$ hadrons. These variables were converted into probabilities $P b_{i}$ for the $\overline{\mathrm{b}}$ hypothesis, and then combined to give the opposite side production tag. This was done in the following way. For each variable a rejection factor $R_{i}$ is defined as $\frac{P b_{i}}{1-P b_{i}}$ and a combined rejection factor $R$ is obtained by taking the product of the rejections $R_{i}$. The combined probability $P$ is then equal to $\frac{R}{1+R}$. In Figure 5 the distribution of the opposite side production tag is shown for 1992-2000 data and simulation. The tagging purity is defined as the fraction of correct flavour assignments at $100 \%$ efficiency, i.e. all events were classified if the cut on the combined probability was set at 0.5 . A purity equal to $68 \%$ has been measured on 1992-2000 simulated events.

A same side production tag is also defined using the fragmentation tracks accompanying the decaying $\mathrm{B}$ meson. Both leading fragmentation pions and kaons are sensitive to the $\mathrm{b}$ or $\overline{\mathrm{b}}$ production flavour. The following quantity $Q_{\text {same }}$ was defined:

$$
Q_{\text {same }}=\sum R\left(p_{i L}, h_{i}\right)\left(1-P_{i}\right) q_{i}
$$

where $h_{i}$ is equal to 1 for a heavy (proton, kaon) or to 0 for a light (electron, muon or pion) particle and the sum extends over all tracks. The parametrization of the function $R-\mathrm{a}$ polynome as a function of $p_{i L}$ - was obtained using simulated events. The variable $Q_{\text {same }}$ was converted into a probability and then combined with the opposite side production tag to give the combined production tag $P_{\text {prod }}$.

In Figure 5 the distribution of $P_{\text {prod }}$ is shown for 1992-2000 data and simulation. The uds and charm quark contributions are small (see Table 2) and are included in the 
total distribution. The tagging purity for $\mathrm{B}_{s}^{0}$ mesons is equal to $71 \%$ for the $1992-2000$ simulation. As expected, this value is higher than the result, $64 \%$, obtained using the opposite side production alone [4]. The difference between data and simulation for the combined production tag, which is apparent in Fig. 5 will be taken into account by fitting the tagging purity for the data (see section 2.6).

\section{2-2000 data}
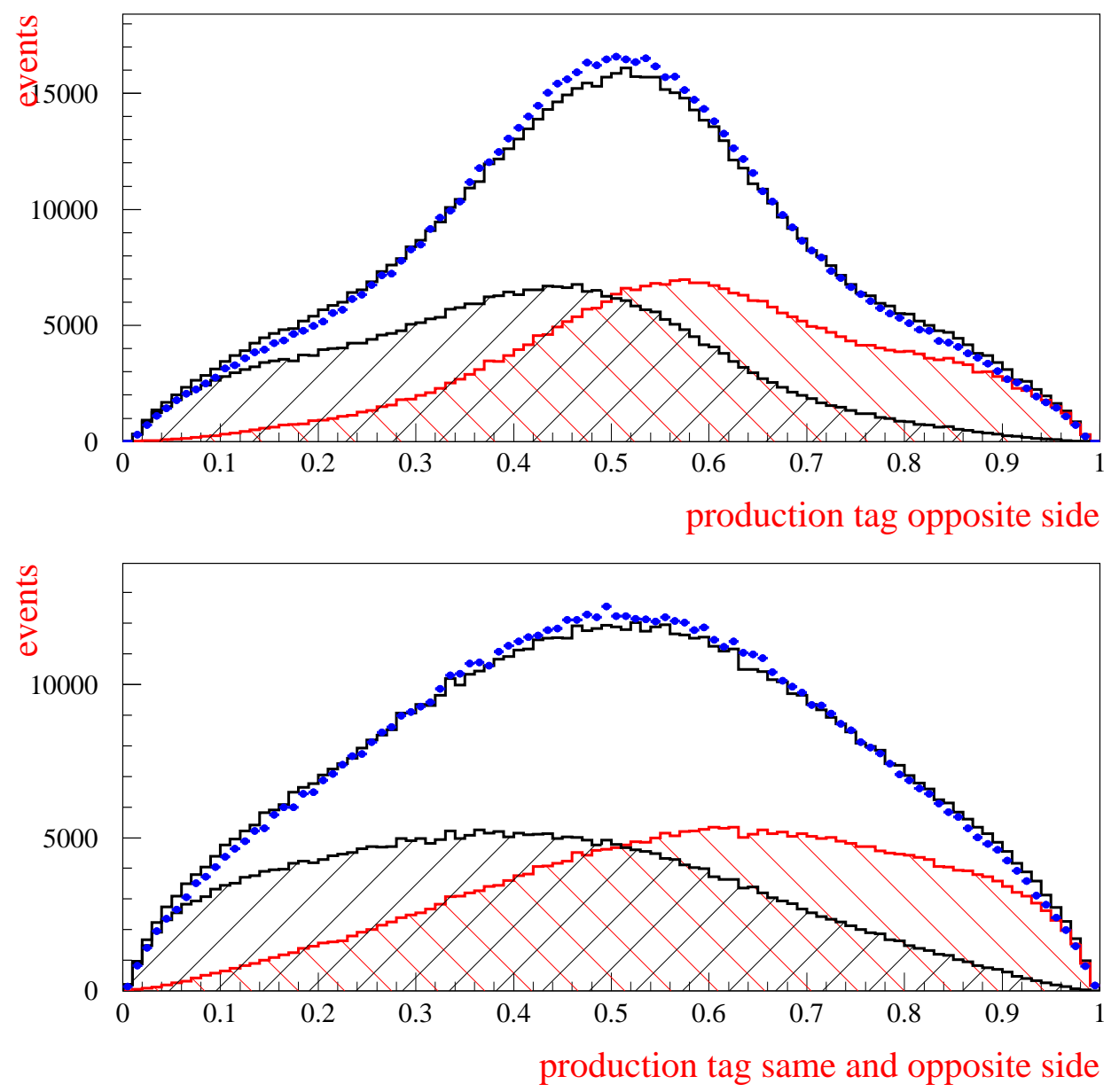

Figure 5: Production tag using only information from the opposite side (upper diagram) and the production tag using information from both sides (lower diagram). The dots correspond to the 1992 to 2000 data, the solid line to the simulation. The hatched areas correspond to the $\mathrm{b}$ (left) and $\overline{\mathrm{b}}$ (right) contributions.

The other important variable in the analysis is the decay tag. In the soft lepton sample this tag is relatively straightforward using the charge of the lepton. Most of the $\mathrm{B}-\overline{\mathrm{B}}$ separation comes from the momentum $p^{\star}$ of the lepton in the $\mathrm{B}$ rest frame that allows the separation of a prompt lepton coming from the B vertex from a lepton coming from a charm decay. Other information in the event (such as, for example, the impact parameter of the lepton with respect to the secondary vertex and the isolation of the lepton (presence of other tracks from charm decay vertex)) helps to improve the $\mathrm{B}-\overline{\mathrm{B}}$ separation. Finally, also the decay tag developed for the inclusive vertex sample (discussed below) was added to improve the performance slightly. 
For events with no lepton, obtaining a decay tag is more difficult. The following approach was taken. All the charged and neutral particles were boosted back in the $\mathrm{B}$ reference frame using the estimated B momentum and direction (see section 2.2). The B-thrust axis was determined in the B reference frame using charged and neutral particles with $P_{i}$ greater than 0.5. The particles were assigned to the forward or backward hemisphere. Usually one hemisphere contains most of the tracks from the B vertex while the other contains most of the tracks from charm decay. This is called a dipole, as the $\mathrm{B}_{s}^{0}$ decays to a $\mathrm{D}_{s}^{-(\star)}$ and a virtual $\mathrm{W}^{+}$and the charge difference between the two hemispheres is equal to \pm 2 . Under the hypothesis that the forward (backward) hemisphere contains the particles from the charm decay and the backward (forward) hemisphere the particles from the $\mathrm{B}$ vertex, the flavour probability of the decaying $\mathrm{B}_{s}^{0}$ is evaluated. This is achieved by using the charge and the momentum $p^{\star}$ in the $\mathrm{B}$ rest frame of the heavy $(p, K)$ and light $(e, \mu, \pi)$ particles. For these parametrizations, the simulation was used. Then a hemisphere probability is evaluated for the hypothesis that the charmed particle is in the forward (backward) hemisphere. This probability depends on the lifetime-signed impact parameter of the tracks with respect to the secondary vertex, on their momenta in the B rest frame and on the hemisphere multiplicity. By combining the hemisphere probability with the flavour probability, the decay tag for the inclusive vertex sample was obtained. The tag was optimized for $\mathrm{B}_{\mathrm{s}}$ mesons.

In Figure 6 the performance of the decay tag $P_{\text {decay }}$ for the soft lepton sample is shown for 1992-2000 data and simulation. The uds and charm quark contributions are small (see Table 2) and not shown explicitly. The tagging purity is $69 \%$ at $100 \%$ efficiency. The events with $P_{\text {decay }}$ from 0 to 0.02 and 0.98 to 1 are due to prompt B decays with a high $p^{\star}$ value. The performance of the decay tag for the inclusive vertex sample is also shown. The $\mathrm{B}_{s}^{0}$ tagging purity is $58 \%$ at $100 \%$ efficiency. The difference between data and simulation for the decay tag will be taken into account by fitting the purity for the data, as is discussed in section 2.6.

\subsection{Sample composition}

For the sample composition the following B-hadron production fractions were assumed $[10]: f_{\mathrm{B}_{\mathrm{s}}}=0.097 \pm 0.011, f_{\mathrm{B} \text { baryons }}=0.103 \pm 0.017, f_{\mathrm{B}_{\mathrm{u}}}=f_{\mathrm{B}_{\mathrm{d}}}=0.40$. For the lifetime of the different $\mathrm{B}$ species it was assumed that [10]: $\tau_{\mathrm{B}_{\mathrm{u}}}=1.65 \mathrm{ps}, \tau_{\mathrm{B}_{\mathrm{d}}}=\tau_{\mathrm{B}_{\mathrm{s}}^{0}}=1.55 \mathrm{ps}$ and $\tau_{\mathrm{B}_{\text {baryons }}}=1.20 \mathrm{ps}$.

Using the simulation, the uds and charm backgrounds were extracted. The background fractions for the different data sets and vertex categories are listed in Table 2 , where $f_{u d s}$ is defined as the number of uds events divided by the total number of events in the sample.

\begin{tabular}{|c|c|c|c|c|c|c|c|c|c|}
\hline background & data set & cat 1 & cat 2 & cat 3 & cat 4 & cat 5 & cat 6 & cat 7 & cat 8 \\
\hline$f_{\text {uds }}$ & $1992-1993$ & .0074 & .0158 & .0288 & .0495 & .0226 & .0407 & .0717 & .1237 \\
$f_{\text {uds }}$ & $1994-2000$ & .0046 & .0076 & .0117 & .0229 & .0138 & .0199 & .0329 & .0588 \\
$f_{\text {charm }}$ & $1992-1993$ & .0202 & .0653 & .1116 & .1779 & .0359 & .0920 & .1433 & .1900 \\
$f_{\text {charm }}$ & $1994-2000$ & .0356 & .0673 & .1201 & .1919 & .0436 & .0928 & .1514 & .2004 \\
\hline
\end{tabular}

Table 2: The background fractions for the 1992-2000 data sets divided according to the different vertex categories. 


\section{2-2000 data}
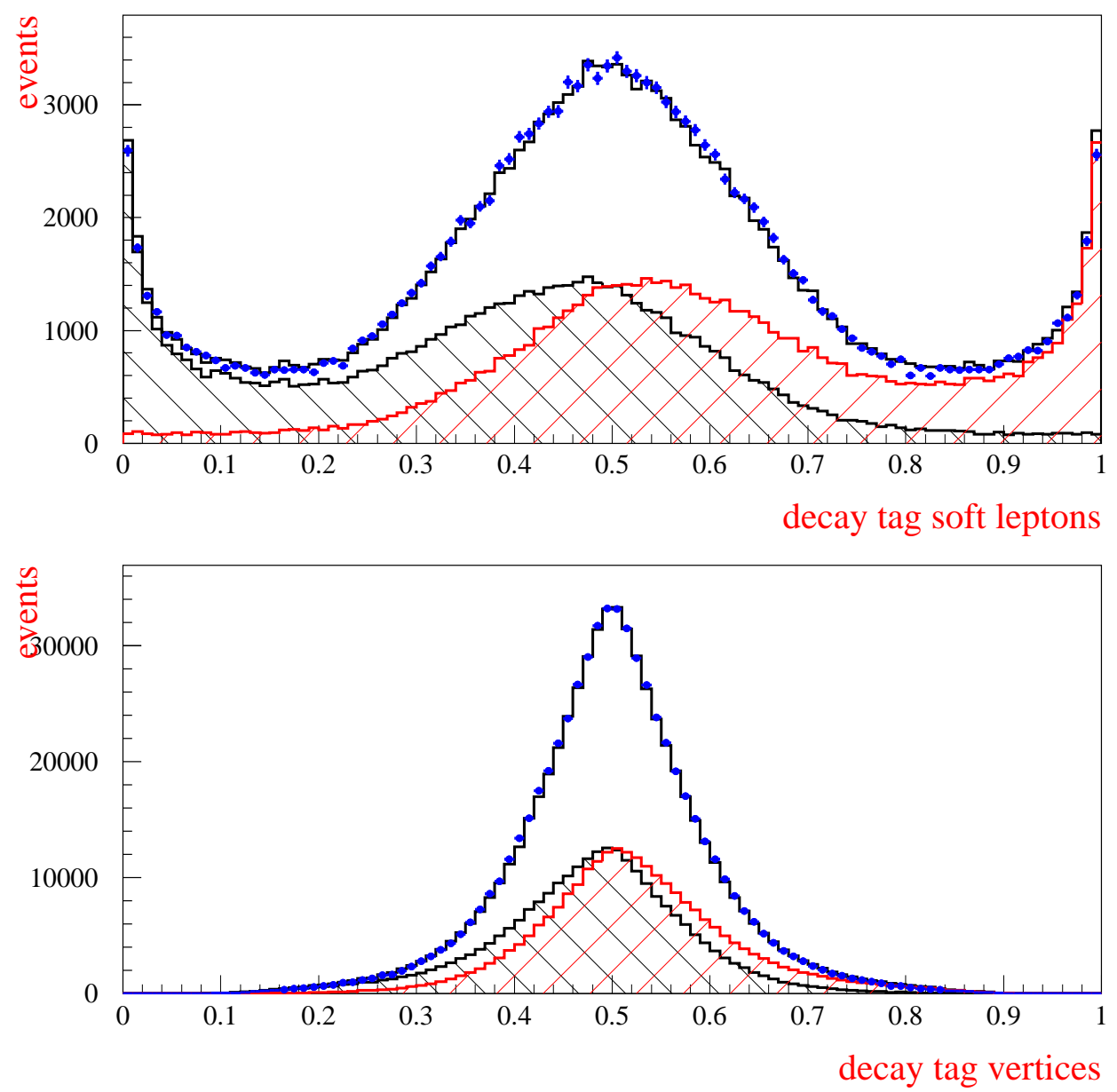

Figure 6: Decay tag for the soft lepton sample (upper diagram) and inclusive vertex sample (lower diagram). The dots correspond to the 1992 to 2000 data, the solid line to the simulation. The hatched areas correspond to the b (right) and $\bar{b}$ (left) contributions at the time of decay.

\subsection{Fitting programme}

The fitting programme provided an analytic description of the data for the like- and unlike-sign tagged events. It was used to fit the amplitude of $\mathrm{B}_{s}^{0}-\overline{\mathrm{B}_{s}^{0}}$ oscillations. In the fitting program the time resolution function $\mathcal{R}\left(t_{\text {rec }}-t_{\text {true }}, t_{\text {true }}\right)$ was parametrized. The resolution function gives the probability that, given a certain value for the true proper time $t_{\text {true }}$, a proper time value $t_{r e c}$ is reconstructed. Two asymmetric Gaussian distributions ${ }^{4}$ are used to describe the main signal, as well as one asymmetric Gaussian to describe poorly measured events and one Gaussian to describe the probability that the secondary vertex is reconstructed near to the primary vertex. The widths of the Gaussian distributions are of the form $\sigma=\sqrt{\sigma_{0}^{2}+\sigma_{p}^{2} t_{\text {true }}^{2}}$ with $\sigma_{p}$ being the relative momentum resolution. The relative normalizations of the Gaussian distributions are left free to vary and parametrized as a constant plus a term proportional to $1-e^{-t_{\text {true }} / \tau}$, where $\tau$ is the

\footnotetext{
${ }^{4}$ The asymmetric Gaussian has two widths, one for proper time values above the central value, the other for below.
} 
average b lifetime. For each vertex category the time resolution function was fitted and the result of the fit is shown in Figure 3. The effect of different parametrisations of the resolution function was found to be neglible with respect to the effect of a change in the proper time resolution (see section 2.2).

The probability $\mathcal{P}_{b}\left(t_{r e c}\right)$ for a B event to be observed at a proper time $t_{r e c}$ was written as a convolution of an exponential B decay distribution with lifetime $\tau$, an acceptance function $A(t)$ and the resolution function:

$$
\mathcal{P}_{b}\left(t_{\text {rec }}\right)=\int_{0}^{\infty} A(t) \mathcal{R}\left(t_{\text {rec }}-t, t\right) \frac{e^{-t / \tau}}{\tau} d t .
$$

The acceptance function was parametrized for the different vertex categories using the simulation. The difference in acceptance for the different B species was found to be negligible. Due to the requirements on the flight distance in the track selection, the acceptance is a smooth, but not flat, function of the proper time. The probabilities for uds $\left(\mathcal{P}_{u d s}\right)$ and charm $\left(\mathcal{P}_{c}\right)$ events for the different vertex categories are parametrized as a function of $t_{r e c}$ with exponential functions whose slopes are determined using the simulation.

Like- or unlike-sign tagged events are those events for which $P_{\text {comb }}$ is respectively larger or smaller than $50 \%$. The combined tagging probability $P_{\text {comb }}$ is defined as

$$
P_{\text {comb }}=P_{\text {prod }} P_{\text {decay }}+\left(1-P_{\text {prod }}\right)\left(1-P_{\text {decay }}\right) .
$$

The tagging purity $\epsilon_{\mathrm{B}_{\mathrm{q}}}$ is expressed in terms of the combined tagging probability $P_{\text {comb }}$. For $\mathrm{B}_{s}^{0}$ events it is given by:

$$
\epsilon_{\mathrm{B}_{\mathrm{s}}}=0.5+\left|P_{\text {comb }}-0.5\right|
$$

The tagging purities for the other $\mathrm{B}$ particles and for the charm and light quark background events were also expressed as functions of $P_{\text {comb }}\left(P_{\text {prod }}\right.$ and $\left.P_{\text {decay }}\right)$ using the simulation (see section 2.6).

The total probability to observe a like-sign tagged event at the reconstructed proper time $t_{r e c}$ is:

$$
\begin{array}{r}
\mathcal{P}^{\text {like }}\left(t_{r e c}\right)=f_{b} \sum_{q=d, s} f_{\mathrm{B}_{\mathrm{q}}} \epsilon_{\mathrm{B}_{\mathrm{q}}} \mathcal{P}_{\text {rec. } \mathrm{B}_{\mathrm{q}}}^{\text {mix. }}\left(t_{\text {rec }}\right)+f_{b} \sum_{q=u, d, s, \text { baryons }} f_{\mathrm{B}_{\mathrm{q}}}\left(1-\epsilon_{\mathrm{B}_{\mathrm{q}}}\right) \mathcal{P}_{\text {rec. } \mathrm{B}_{\mathrm{q}}}^{\text {unmix. }}\left(t_{\text {rec }}\right)+ \\
f_{c}\left(1-\epsilon_{c}\right) \mathcal{P}_{c}\left(t_{\text {rec }}\right)+f_{u d s}\left(1-\epsilon_{u d s}\right) \mathcal{P}_{\text {uds }}\left(t_{\text {rec }}\right)
\end{array}
$$

and correspondingly for an unlike-sign tagged event:

$$
\begin{aligned}
& \mathcal{P}^{\text {unlike }}\left(t_{r e c}\right)=f_{b} \sum_{q=d, s} f_{\mathrm{B}_{\mathrm{q}}}\left(1-\epsilon_{\mathrm{B}_{\mathrm{q}}}\right) \mathcal{P}_{\text {rec. } \mathrm{B}_{\mathrm{q}}}^{\text {mix. }}\left(t_{r e c}\right)+f_{b} \sum_{q=u, d, s, \text { baryons }} f_{\mathrm{B}_{\mathrm{q}}} \epsilon_{\mathrm{B}_{\mathrm{q}}} \mathcal{P}_{\mathrm{B}_{\mathrm{q}}}^{\text {unmix. }}\left(t_{\text {rec }}\right)+ \\
& f_{c} \epsilon_{c} \mathcal{P}_{c}\left(t_{r e c}\right)+f_{u d s} \epsilon_{u d s} \mathcal{P}_{u d s}\left(t_{r e c}\right) .
\end{aligned}
$$

For the mixed $\mathrm{B}_{d}^{0}$ and $\mathrm{B}_{s}^{0}$ mesons one has the following expression $(q=d, s)$ :

$$
\mathcal{P}_{\text {rec. } \mathrm{B}_{\mathrm{q}}}^{\text {mix. }}\left(t_{\text {rec }}\right)=\int_{0}^{\infty} A(t) \mathcal{R}\left(t_{r e c}-t, t\right) \mathcal{P}_{\mathrm{B}_{\mathrm{q}}}^{\text {mix. }}(t) d t,
$$

while for the unmixed case the $\mathrm{B}_{u}$ and $\mathrm{B}$ baryons also have to be included $(q=$ $u, d, s$,baryons):

$$
\mathcal{P}_{\text {rec. } \mathrm{B}_{\mathrm{q}}}^{\text {unmix. }}\left(t_{\text {rec }}\right)=\int_{0}^{\infty} A(t) \mathcal{R}\left(t_{\text {rec }}-t, t\right) \mathcal{P}_{\mathrm{B}_{\mathrm{q}}}^{\text {unmix. }}(t) d t,
$$

where $\mathcal{P}_{\mathrm{B}_{\mathrm{q}}}^{(\text {un)mix. }}(t)$ are defined in Eqs. (1) and (2). 


\subsection{Modelling the simulation and data}

The present analysis uses the production and decay probabilities on an event-by-event basis. The tagging purity $\epsilon_{\mathrm{B}_{\mathrm{s}}}$ is calculated from these quantities as defined in Eq. (12). The production and decay probabilities - and thus the tagging purities - for the different B species, charm and light quarks are different. These differences have to be parametrized in the analytic fitting programme. For this simulated events were used.

The new parametrization is obtained by modifying the probability $P\left(P_{\text {prod }}\right.$ or $\left.P_{\text {decay }}\right)$. For this purpose a parameter $\alpha$ is introduced and the new probability is defined as:

$$
P_{\text {new }}=R^{\alpha} /\left(1+R^{\alpha}\right),
$$

where the rejection $R$ is defined as $\frac{P}{1-P}$. A parameter value of 1 means that the probability remains unchanged. It was found out on simulation that this particular parametrisation gives an accurate description of the tag performance for neutral B species. For example the $\alpha$ parameter for a $\mathrm{B}$ hadron is obtained in the following way. Using the simulation the distribution of the probability $P$ is plotted separately for $\mathrm{B}$ and $\overline{\mathrm{B}}$ hadrons. The two distributions are divided and fitted to the expression (17) leaving free the $\alpha$ parameter. This procedure is illustrated in Fig. 7 using the decay tag for the inclusive vertex sample for the $\mathrm{B}_{\mathrm{d}}$ meson.

For leptons, the decay purities for the different B species were studied on simulation and found to be very similar. The decay tag parameter for the soft lepton sample was therefore put equal to 1 . The decay tag parameters for the inclusive vertex sample and soft lepton sample as well as the production tag parameter are listed in Table 3. The values are obtained from the simulation. For the charm quark a parameter $\alpha_{D}$ of 4.2 is used if the probability lies between 0.2 and 0.8 , otherwise $\alpha_{D}=1$. For the soft lepton sample the relatively high value of $\alpha_{D}$ of 4.2 is understandable, because a lepton coming from the charmed particle, at relatively low $p^{\star}$, will tag correctly the charge of the charmed particle. Note that the parameters $\alpha_{D}$ and $\alpha_{P}$ for the different $\mathrm{B}$ species are quite similar, except for the $\mathrm{B}_{u}$ where $\alpha_{D}$ varies as a function of $P^{5}$ between 0.75 and 1 . For this reason, tagging purities for $\mathrm{B}_{u}$ and for the other types of $\mathrm{b}$ hadrons have been controlled directly from the data, as explained in the following. From the new probability $P_{\text {new }}^{\mathrm{B}_{\mathrm{q}}, \mathrm{uds}, \mathrm{c}}$, the combined probability $P_{c o m b}^{\mathrm{B}_{\mathrm{q}}, \mathrm{uds}, \mathrm{c}}$ is calculated using Eq. (11) and the purity $\epsilon_{\mathrm{B}_{\mathrm{q}}, \mathrm{uds}, \mathrm{c}}$ is obtained using:

$$
\epsilon_{\mathrm{B}_{\mathrm{q}}, \mathrm{uds}, \mathrm{c}}=0.5+\left|P_{c o m b}^{\mathrm{B}_{\mathrm{q}}, \mathrm{uds}, \mathrm{c}}-0.5\right| .
$$

\footnotetext{
${ }^{5}$ The functional form used is $\alpha=\alpha_{0}+\alpha_{1} e^{2 \mid P}-0.5 \mid$.
}

\begin{tabular}{|c|c|c|c|}
\hline Particle & $\begin{array}{c}\text { inclusive vertex sample } \\
\text { decay tag } \alpha_{D}\end{array}$ & $\begin{array}{c}\text { soft lepton sample } \\
\text { decay tag } \alpha_{D}\end{array}$ & $\begin{array}{c}\text { all events } \\
\text { production tag } \alpha_{P}\end{array}$ \\
\hline $\mathrm{B}_{\mathrm{s}}$ & 1 & 1 & 1 \\
$\mathrm{~B}_{\mathrm{d}}$ & 1.15 & 1 & 1.13 \\
$\mathrm{~B}_{\mathrm{u}}$ & 0.75 to & 1 & 0.3 to 0.8 \\
B baryon & 0.80 & 1 & 1.09 \\
uds & 0.20 & 0.20 & 0.80 \\
charm & $4.2(\mathrm{P}=0.2-0.8)$ & $4.2(\mathrm{P}=0.2-0.8)$ & 0.50 \\
\hline
\end{tabular}

Table 3: The parameters $\alpha$ for the production and decay tag for the different particles as obtained from simulation. 

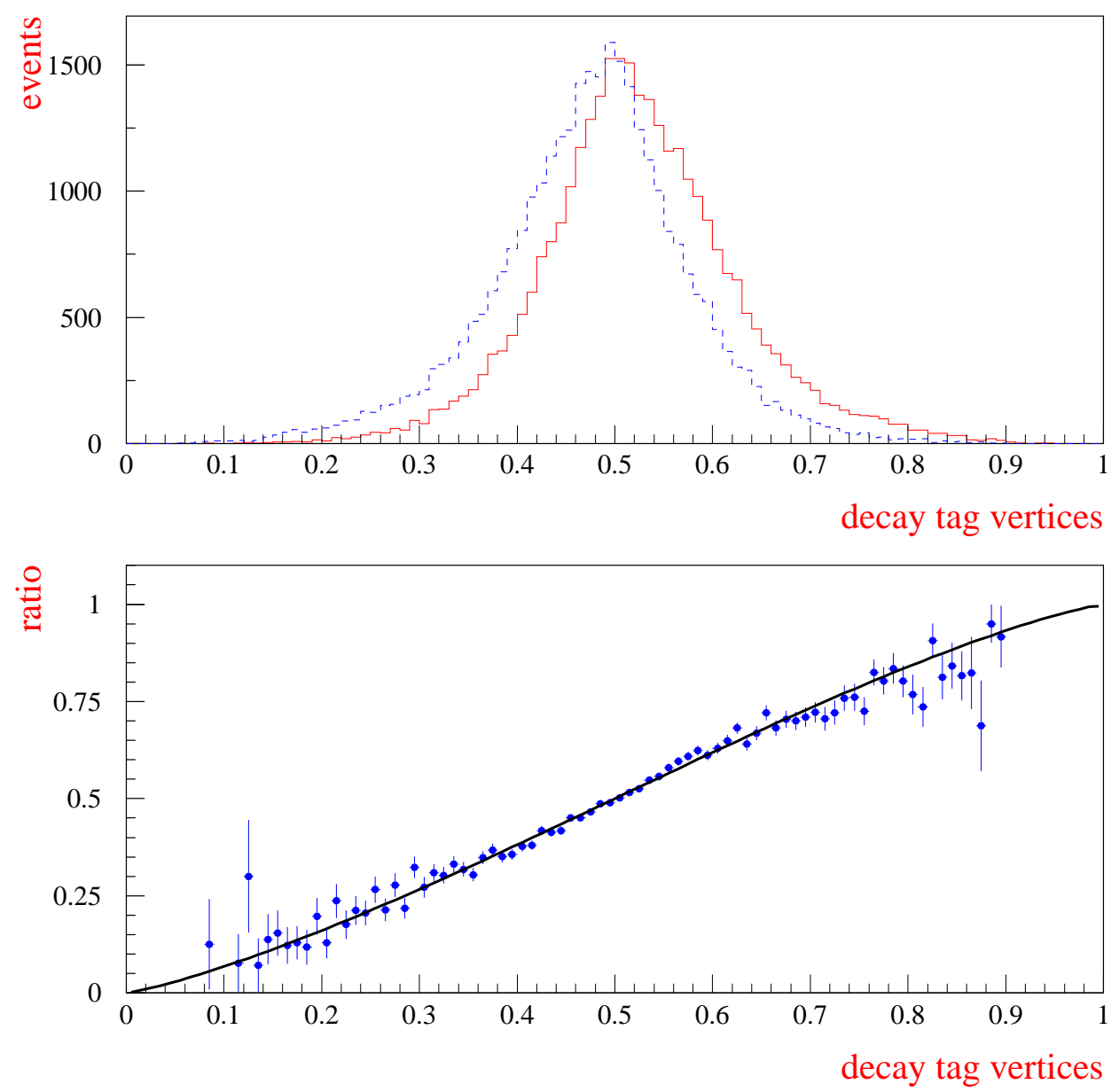

Figure 7: Distributions for the decay tag for the inclusive vertex sample for the $B_{d}$ (solid line) and $\overline{\mathrm{B}_{\mathrm{d}}}$ (dashed line) mesons. The bottom plot shows the ratio of the two distributions with a fit of Eq.(17) giving the $\alpha$ parameter of 1.15.

It is important also to have a correct modelling of the tagging purity for the data i.e. to have a good description of the like- and unlike-sign tagged events. Using the data for each category, a correction factor $C$ to the parameter $\alpha$ is fitted:

$$
\alpha_{D}^{\text {data }}=C \alpha_{D}
$$

where $C$ is determined from the fraction of like-sign tagged events. The $C$ factor was determined iteratively and $\Delta m_{d}=0.531$ (see section 2.7) was finally used. For the soft lepton sample, the results are shown in Table 4.

The 1992-1993 and 1994-2000 data sets have different performance for tracking and lepton identification and therefore the fitted $C$ values can be different. The total error on $C$ for the soft lepton sample is better than $\pm 5 \%$.

The parameter $\alpha$ for the decay tag in the inclusive vertex sample for a $\mathrm{B}_{u}$ meson is different - it also varies as a function of the tagging probability -from those for the other $\mathrm{B}$ particles (see Table 3). By separating the inclusive vertex sample into one enriched and one depleted in $\mathrm{B}_{u}$ particles it was possible to determine, from the data, the correction 


\begin{tabular}{|c|c|c|}
\hline data set & category & fitted value for $C$ \\
\hline $1992-1993$ & 1 & $0.95 \pm 0.05$ \\
& 2 & $0.81 \pm 0.05$ \\
& 3 & $0.76 \pm 0.05$ \\
& 4 & $0.82 \pm 0.05$ \\
\hline $1994-2000$ & 1 & $0.77 \pm 0.04$ \\
& 2 & $0.69 \pm 0.04$ \\
& 3 & $0.83 \pm 0.04$ \\
& 4 & $0.84 \pm 0.05$ \\
\hline
\end{tabular}

Table 4: The fitted correction factor $C$ for the soft lepton sample.

factor $C$ for $\mathrm{B}_{u}$ mesons and for the other particles. These samples were obtained by cutting on the secondary vertex charge. Three fits were performed. First it was assumed that the correction factors $C$ for all types of B particles were identical. Then in a second fit it was assumed that $C$ for the non- $\mathrm{B}_{u}$ particles was equal to 1 and the value of the correction factor $C$ for the $\mathrm{B}_{u}$ particles was fitted. From the $\chi^{2}$ of the fit it was clear that the second fit result was preferred. The value of the correction factor $C$ for $\mathrm{B}_{u}$ particles was fixed to the average value between the first and second fit results and a third fit was performed leaving $C$ free for non- $B_{u}$ particles. The results for the final fit are shown in Table 5, where the errors quoted in the third column correspond to the statistical errors obtained in the first fit. If another procedure was chosen a different $C$ value would

\begin{tabular}{|c|c|c|c|}
\hline data set & category & $\begin{array}{c}\text { correction factor } C \\
\text { for } \mathrm{B}_{d}, \mathrm{~B}_{s} \text { and } \mathrm{B}_{\text {baryon }}\end{array}$ & $\begin{array}{c}\text { correction factor } C \\
\text { for } \mathrm{B}_{u} \text { mesons }\end{array}$ \\
\hline 1992-1993 & 5 & $0.75 \pm 0.07$ & 0.54 \\
& 6 & $0.76 \pm 0.06$ & 0.54 \\
& 7 & $0.72 \pm 0.07$ & 0.40 \\
& 8 & $0.63 \pm 0.09$ & 0.20 \\
\hline $1994-2000$ & 5 & $0.93 \pm 0.05$ & 0.60 \\
& 6 & $0.94 \pm 0.04$ & 0.60 \\
& 7 & $0.83 \pm 0.07$ & 0.40 \\
& 8 & $0.63 \pm 0.09$ & 0.20 \\
\hline
\end{tabular}

Table 5: The fitted correction factors $C$ for the inclusive vertex sample.

have been obtained. The largest change in the $C$ value for non- $\mathrm{B}_{u}$ particles is quoted as a systematic error and amounts to $\pm 15 \%$. The systematic error is larger than the statistical error.

It was found, using the simulation, that the acceptance for the uds and charm quarks depends on the tagging purity. The acceptance $A(t)$ for $\mathrm{B}$ events also varies slightly as a function of the tagging purity. This was taken into account in the like- and unlike-sign probability distributions. A comparison between data and simulation showed a slightly different acceptance function. The acceptance function was corrected to obtain better agreement between the data and the parametrisation in the fitting program. Note that for $\mathrm{B}_{d}^{0}-\overline{\mathrm{B}_{d}^{0}}$ and $\mathrm{B}_{s}^{0}-\overline{\mathrm{B}_{s}^{0}}$ oscillations, only the fraction of like-sign tag events is relevant and to first order the acceptance correction drops out. 
In Figure 8 the distributions for the like- and unlike-sign tagged events, as a function of the proper time, corresponding respectively to the soft lepton sample and to the inclusive vertex sample, are shown. In these Figures, the events have been weighted by $\left|\epsilon_{\mathrm{B}_{\mathrm{s}}}-0.5\right|$. In this way events with a higher tagging purity acquire a higher weight. Events with a purity of 0.5 carry no information and have a weight equal to zero. A good description of the data is obtained.

\section{4-2000 data}
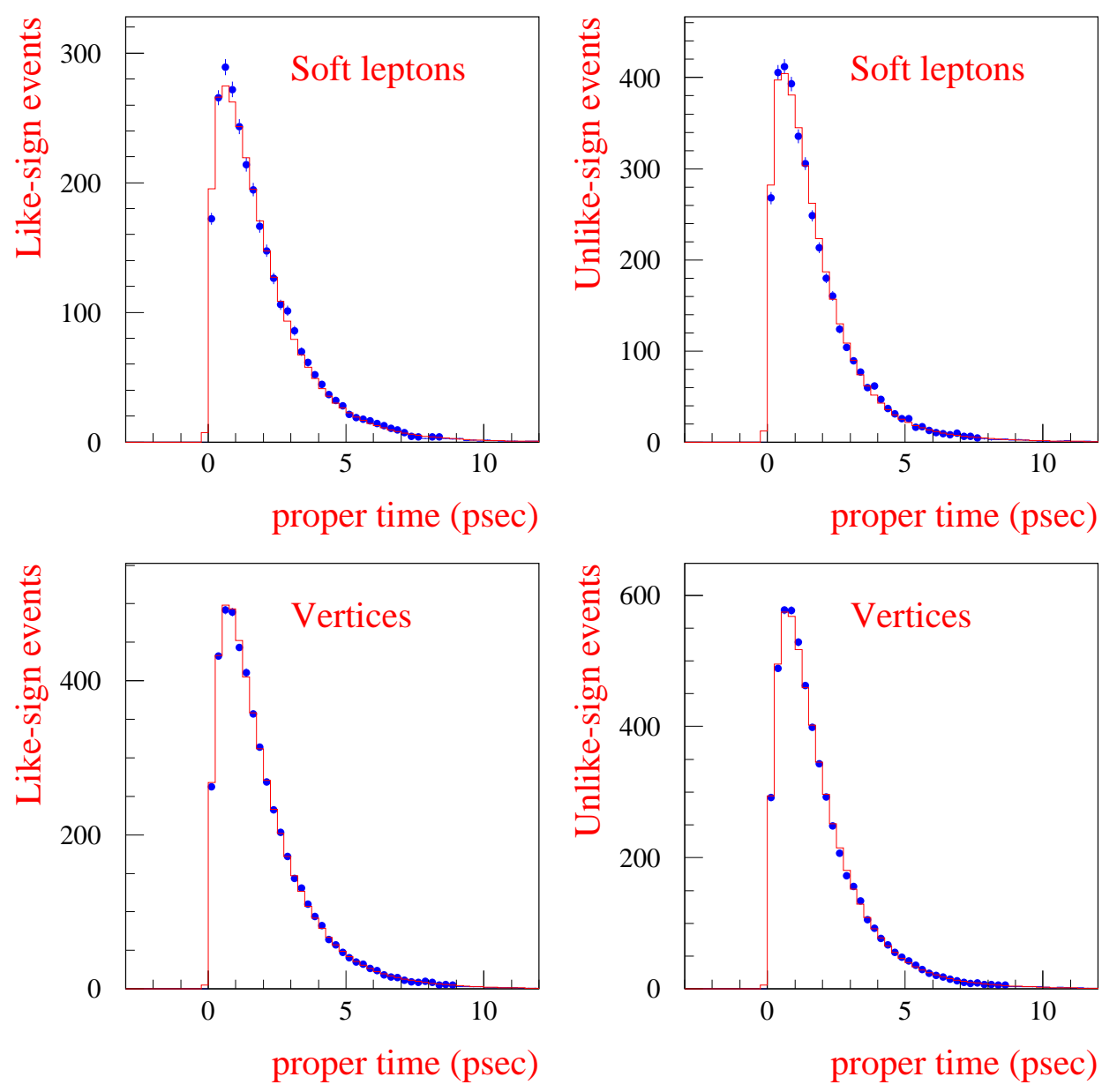

Figure 8: Reconstructed proper time distributions for the like- and unlike-sign tagged events corresponding to the soft lepton (upper diagram) and inclusive vertex (lower diagram) samples. The 1994 to 2000 data are shown as dots, the fitted parametrization is shown as a histogram.

In Figures 9 and 10 the fractions of weighted - as described above - like-sign tagged events, as a function of the proper time, for the soft lepton sample and inclusive vertex sample, are shown for the 1992 to 2000 data. In these Figures, values of $\Delta m_{d}$ of $0.495 \mathrm{ps}^{-1}$ and $\Delta m_{s}$ of $15 \mathrm{ps}^{-1}$ are used in the parametrizations corresponding to the continuous lines. 
1992-1993 data
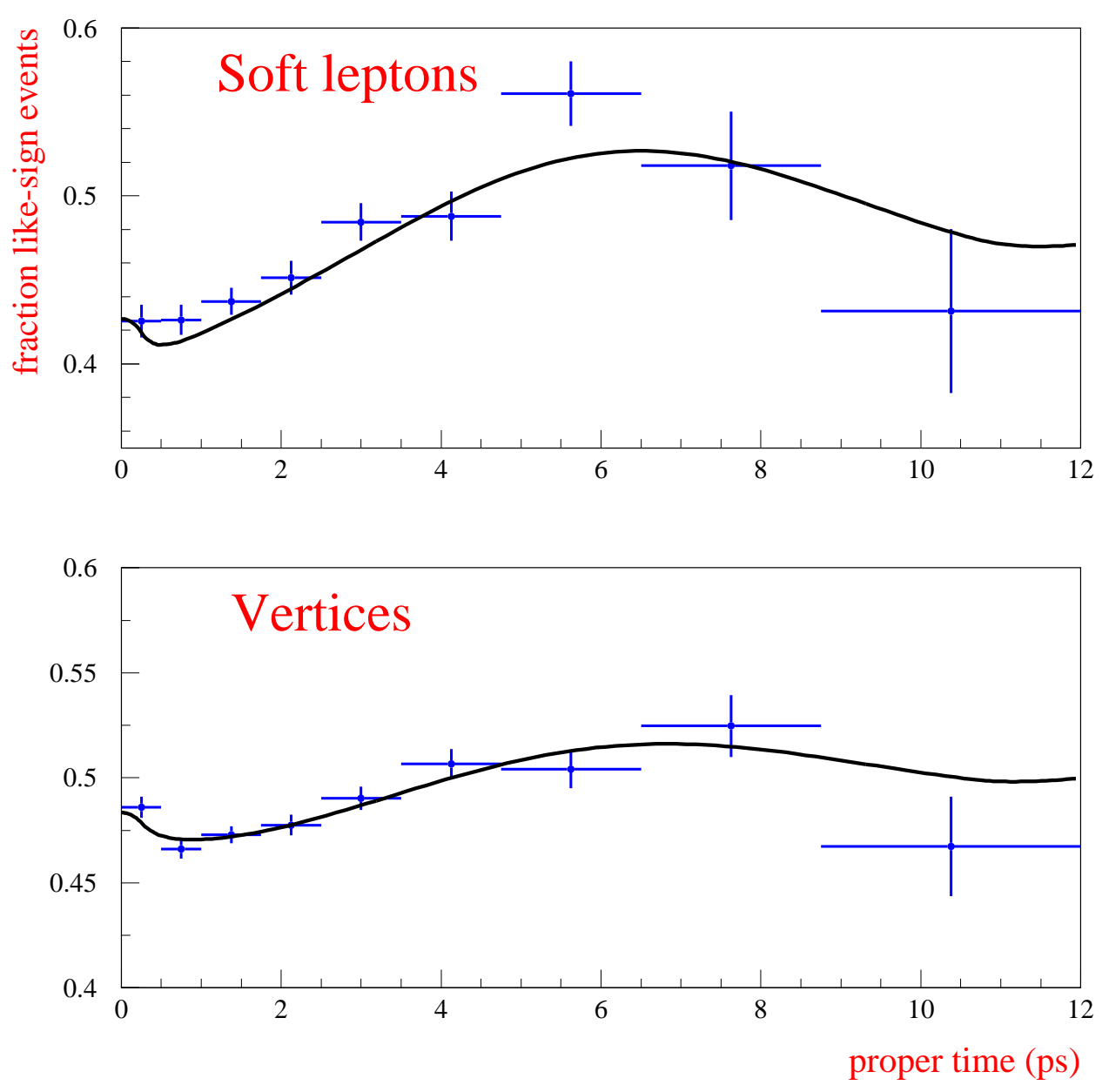

Figure 9: Fraction of like-sign tagged events as a function of the reconstructed proper time for the soft lepton and inclusive vertex samples. The 1992 and 1993 data are shown as points with error bars, the parametrization is given as a solid line. 


\section{4-2000 data}
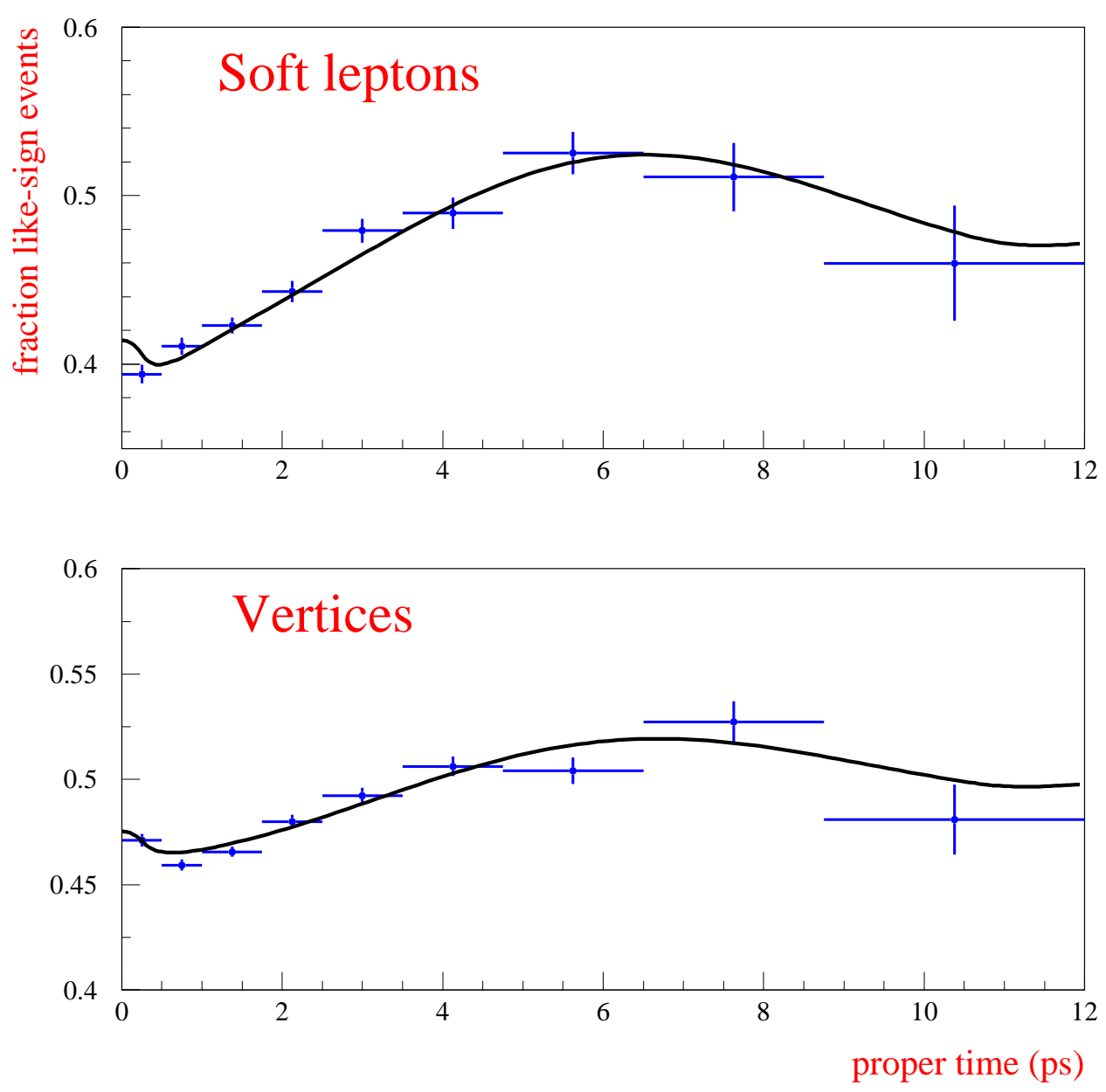

Figure 10: Fraction of like-sign tagged events as a function of the reconstructed proper time for the soft lepton and inclusive vertex samples. The 1994 to 2000 data are shown as points with error bars, the parametrization is given as a solid line. 


\subsection{Measurement of the $\mathrm{B}_{d}$ oscillation frequency}

The mass difference between the two physical states in the $\mathrm{B}_{d}^{0}-\overline{\mathrm{B}_{d}^{0}}$ system was determined by fitting the fraction of weighted like-sign tagged events - shown in Figures 9, 10 - as a function of the reconstructed proper time $t$. The following expression was used for the number of weighted like-sign events:

$$
\begin{aligned}
& N_{\text {like }}(t)=A(t) N_{b} f_{\mathrm{B}_{\mathrm{u}}} \frac{e^{-t / \tau_{\mathrm{B}_{\mathrm{u}}}}}{\tau_{\mathrm{B}_{\mathrm{u}}}}\left(1-\epsilon_{b}\right) \\
& +A(t) N_{b} f_{\mathrm{B}_{\text {baryon }}} \frac{e^{-t / \tau_{\mathrm{B}} \text { baryon }}}{\tau_{\mathrm{B}_{\text {baryon }}}}\left(1-\epsilon_{b}\right) \\
& +A(t) N_{b} f_{\mathrm{B}_{\mathrm{s}}} \frac{e^{-t / \tau_{\mathrm{B}_{\mathrm{s}}}}}{2 \tau_{\mathrm{B}_{\mathrm{s}}}} \\
& +A(t) N_{b} f_{\mathrm{B}_{\mathrm{d}}} \frac{e^{-t / \tau_{\mathrm{B}}}}{2 \tau_{\mathrm{B}_{\mathrm{d}}}}\left[\left(1-2 \epsilon_{d}\right) \cos \left(\Delta m_{d} t\right)+1\right] \\
& +\left(1-\epsilon_{c}\right) N_{c}(t)+\left(1-\epsilon_{u d s}\right) N_{u d s}(t) \text {. }
\end{aligned}
$$

The total number of weighted events is equal to:

$$
N_{t o t}(t)=\sum A(t) N_{b} f_{\mathrm{Bi}} e^{-t / \tau_{B i}} / \tau_{B i}+N_{c}(t)+N_{u d s}(t)
$$

The event-by-event tagging purity is used as a weight. The values for $f_{\mathrm{Bi}}$ and for the B-hadron lifetimes were fixed at the values listed in section 2.4. $N_{b}$ is the total number of b quark events. The functions $N_{c}(t)$ and $N_{u d s}$ as well as the acceptance $A(t)$ were parametrized using the simulation. The total number of events from charm and uds quarks are obtained by intergrating these functions. The tagging purities $\epsilon_{c}$ and $\epsilon_{u d s}$ were taken from the simulation.

The like-sign tagged fraction $\frac{N_{\text {like }}}{N_{\text {tot }}}$ was fitted in the range from 0.5 to 12 ps using a binned $\chi^{2}$ fit. First a fit was performed on the simulated data, i.e. the parametrization as shown in Figures 9 and 10. In this fit $\Delta m_{d}$, the $\mathrm{B}_{d}$ mass difference, is fixed and $\epsilon_{d}, R_{1}$, $R_{2}$ and $a$ are left free, where $\epsilon_{d}$ is the $\mathrm{B}_{d}$ tagging purity and the tagging purity for the other $\mathrm{b}$ hadrons $\epsilon_{b}$ is parametrized as: $\epsilon_{b}=R_{1} e^{a t}+R_{2} e^{-t}$. The parameter $R_{2}$ takes into account the slight dependence of the tagging purity as a function of the proper time.

In a second fit, the data were fitted leaving free $\Delta m_{d}, \epsilon_{d}, R_{1}$ and $R_{2}$. The parameter $a$ was fixed to the value of $8.510^{-3} \mathrm{ps}^{-1}$ obtained in the previous fit to the simulation. The results for the different parameters are: $\epsilon_{d}=0.575 \pm 0.009(0.579), R_{1}=0.550 \pm 0.005$ (0.554) and $R_{2}=0.080 \pm 0.022(0.059)$. Within parentheses are given the results for the fit to the simulated data.

The result for the $\mathrm{B}_{d}$ mass difference is $\Delta m_{d}=0.531 \pm 0.025$ (stat.) with a $\chi^{2} / n d f$ of 22.5/(23-4), as shown in Figure 11.

The reason for performing a four parameter fit is that both tagging purities for the $\mathrm{B}_{d}^{0}$ meson and for the other B particles are determined using the data. Therefore systematic uncertainties on these parameters were largely reduced. In this way the fit results become also less sensitive to, for example, the fraction of $\mathrm{B}_{s}^{0}$ mesons. Due to the fact that the fit was first applied and tuned to the simulated data, the resolution function is taken into account.

A breakdown of the systematic errors affecting the measurement is given in Table 6 . The range of values for the fractions and lifetimes of the different $\mathrm{B}$ species come from ref. [10]. The fractions of $\mathrm{B}_{s}^{0}$ mesons and $\mathrm{B}$ baryons were changed (correspondingly the other B fractions are recalculated) as well as the lifetimes and backgrounds. The tagging correction factor $C$ (see section 2.6) was varied by a relative $5 \%$ for the soft lepton sample and by $15 \%$ for the inclusive vertex sample. The proper times were scaled with $1 \%$, coming 
Inclusive vertices and soft leptons

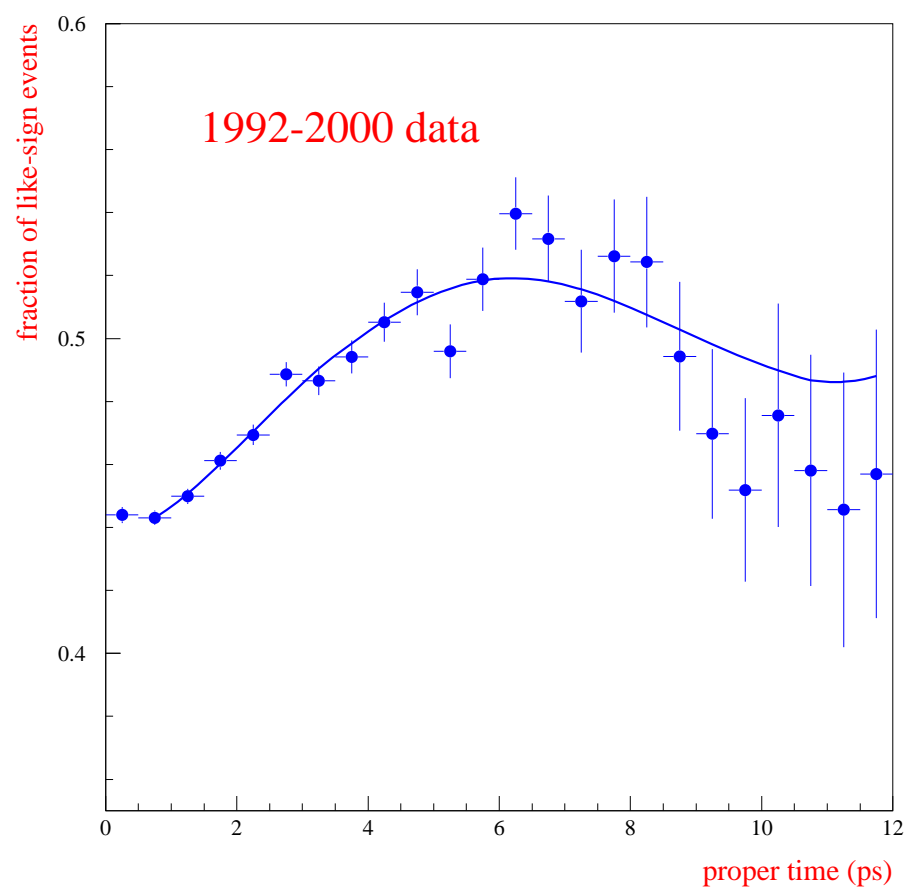

Figure 11: Fraction of like-sign tagged events as a function of the reconstructed proper time using 1992-2000 data. The data were shown as points with error bars, the solid line corresponds to the fit.

from the scale uncertainty on the reconstructed decay length and momentum, and the corresponding systematic error on $\Delta m_{d}$ was $-0.0049 \mathrm{ps}^{-1}$. The resolution function $\mathcal{R}$ (see section 2.5) was smeared by an additional Gaussian term with a width of $0.1+0.03 t \mathrm{ps}$ and the resulting shift in $\Delta m_{d}$ was $0.0037 \mathrm{ps}^{-1}$. This additional smearing corresponds to a $10 \%$ systematic error on the expected decay length resolution and a $10 \%$ systematic error on the expected momentum resolution.

The total systematic error amounts to $0.007 \mathrm{ps}^{-1}$.

The final result is thus:

$$
\Delta m_{d}=(0.531 \pm 0.025 \text { (stat) } \pm 0.007 \text { (syst.) }) \mathrm{ps}^{-1} .
$$

The total error is therefore $0.027 \mathrm{ps}^{-1}$.

This result for the mass difference between the two physical states in the $\mathrm{B}_{d}^{0}-\overline{\mathrm{B}_{d}^{0}}$ system is compatible with those from other experiments [10].

A fit was also performed to extract the width difference $\Delta \Gamma_{B_{d}}$. In the fit, the expression in Eq. (20) $\left[\left(1-2 \epsilon_{d}\right) \cos \left(\Delta m_{d} t\right)+1\right]$ was replaced by $\left[\left(1-2 \epsilon_{d}\right) \cos \left(\Delta m_{d} t\right)+\cosh \left(\Delta \Gamma_{\mathrm{B}_{\mathrm{d}}} t / 2\right)\right]$ and the expression in Eq. (21) was modified accordingly. The result of the five parameter fit is $\left|\Delta \Gamma_{\mathrm{B}_{\mathrm{d}}}\right|=(0.00 \pm 0.06) \mathrm{ps}^{-1}$. The total systematic error was evaluated for the error sources listed in Table 6 and found to be $0.0002 \mathrm{ps}^{-1}$. Using the measured $\mathrm{B}_{d}$ lifetime $\tau_{\mathrm{B}_{\mathrm{d}}}=(1.55 \pm 0.03) \mathrm{ps}[10],\left|\Delta \Gamma_{\mathrm{B}_{\mathrm{d}}}\right| / \Gamma_{\mathrm{B}_{\mathrm{d}}}=0.00 \pm 0.09$ (tot). The following upper limit was derived:

$$
\left|\Delta \Gamma_{\mathrm{B}_{\mathrm{d}}}\right| / \Gamma_{\mathrm{B}_{\mathrm{d}}}<0.18 \text { at } 95 \% \mathrm{CL} \text {. }
$$




\begin{tabular}{|c|c|c|}
\hline error source & values & systematic error $\left(\mathrm{ps}^{-1}\right)$ \\
\hline$f_{\mathrm{B}_{\mathrm{s}}}$ & 0.097 to $0.108[10]$ & -0.00021 \\
$f_{\mathrm{B} \text { baryon }}$ & 0.103 to $0.12[10]$ & 0.00039 \\
$\tau_{\mathrm{B}_{\mathrm{s}}}$ & 1.55 to $1.60 \mathrm{ps}[10]$ & 0.0001 \\
$\tau_{\mathrm{B}_{\mathrm{u}}}$ & 1.65 to $1.67 \mathrm{ps}[10]$ & -0.0008 \\
$\tau_{\mathrm{B}_{\mathrm{d}}}$ & 1.55 to $1.58 \mathrm{ps}[10]$ & 0.0012 \\
$\tau_{\mathrm{B} \text { baryon }}$ & 1.2 to $1.25 \mathrm{ps}[10]$ & -0.0008 \\
$u d s$ background scale factor & 1 to 1.10 & -0.00022 \\
charm background scale factor & 1 to 1.10 & 0.00052 \\
tagging factor $\Delta C / C$ & variation $5 \%(15 \%)$ & 0.0006 \\
scale factor proper time & 1 to 1.01 & -0.0049 \\
resolution smearing & & 0.0037 \\
\hline Total systematic error & \multicolumn{2}{|l}{} \\
\hline
\end{tabular}

Table 6: The systematic errors affecting the $\Delta m_{d}$ measurement.

\subsection{Search for $\mathbf{B}_{s}^{0}-\overline{\mathbf{B}_{s}^{0}}$ oscillations}

To search for $\mathrm{B}_{s}^{0}-\overline{\mathrm{B}_{s}^{0}}$ oscillations a likelihood fit was performed, where the negative log-likelihood is defined as:

$$
\mathcal{L}=-\sum_{\text {like-sign }} \ln \left(\mathcal{P}^{\text {like }}\left(t_{\text {rec }}, P_{\text {comb }}, P_{\text {decay }}\right)\right)-\sum_{\text {unlike-sign }} \ln \left(\mathcal{P}^{\text {unlike }}\left(t_{\text {rec }}, P_{\text {prod }}, P_{\text {decay }}\right)\right)
$$

where the expression for $\mathcal{P}^{\text {like }}$ and $\mathcal{P}^{\text {unlike }}$ can be found in Eqs. (13) and (14). To extract results from this fit the so-called amplitude method was used [11]. For the mixed and unmixed $\mathrm{B}_{s}^{0}$ events the following expressions were used:

$$
\mathcal{P}_{\mathrm{B}_{\mathrm{s}}^{0}}^{\text {unmix. }}=\frac{1}{2 \tau_{\mathrm{B}_{\mathrm{s}}}} e^{-\frac{t}{\tau_{\mathrm{B}_{\mathrm{s}}}}}\left[1+A \cos \left(\Delta m_{s} t\right)\right]
$$

and similarly:

$$
\mathcal{P}_{\mathrm{B}_{\mathrm{s}}^{0}}^{\text {mix. }}=\frac{1}{2 \tau_{\mathrm{B}_{\mathrm{s}}}} e^{-\frac{t}{\tau_{\mathrm{B}_{\mathrm{s}}}}}\left[1-A \cos \left(\Delta m_{s} t\right)\right] .
$$

$\mathrm{B}_{s}$ oscillations will correspond to a value $\mathrm{A}$ of unity. The oscillation amplitude $A$ and its error $\sigma_{A}$ were fitted to the data as a function of $\Delta m_{s}$. The result of the amplitude fit is shown in Figures 12 and 13.

Before discussing the result and its interpretation, the systematic errors have been studied. This was done by changing one parameter at a time (for example $f_{\mathrm{B}_{\mathrm{s}}}$ ) and redoing the full amplitude fit. The systematic error was then evaluated as [11]:

$$
\sigma_{A}^{\text {syst }}=A_{1}-A_{0}+\left(1-A_{0}\right) \frac{\sigma_{A_{1}}^{\text {stat }}-\sigma_{A_{0}}^{\text {stat }}}{\sigma_{A_{1}}^{\text {stat }}},
$$

where $A_{0}\left(A_{1}\right)$ and $\sigma_{A_{0}}\left(\sigma_{A_{1}}\right)$ denote the fitted amplitude and error before (after) changing the parameter. The last term in Eq.(25) takes into account the change in the error of the fitted amplitude. The following parameters have been changed as in Table 6 :

- $f_{\mathrm{B}_{\mathrm{s}}}$ from 0.097 to 0.108 ,

- the $u d s$ and charm backgrounds have been scaled up by $10 \%$,

- the tagging purity has been changed by varying the correction factor $C$ by $5 \%$ for the soft lepton tag and by $15 \%$ for the inclusive vertex tag, 
- the constant term $\sigma_{0}$ for $\mathcal{R}$, the width of the resolution function (see section 2.5), has been changed by a relative $10 \%$,

- $\sigma_{p}$, the width of the resolution function for the momentum, has been changed by a relative $10 \%$.

The total systematic error as a function of $\Delta m_{s}$ is shown in Figure 12b. It is at most $35 \%$ of the statistical error.

Using the results for the amplitude and its error it is possible to obtain the $95 \% \mathrm{CL}$ exclusion region or sensitivity. This region corresponds to $A+1.645 \sigma_{A}<1$. This curve is shown in Figures 12 and 13. No $\mathrm{B}_{s}^{0}-\overline{\mathrm{B}_{s}^{0}}$ oscillations have been observed in the data. A limit on the mass difference of the two physical $\mathrm{B}_{s}^{0}$ states can be put:

$$
\Delta m_{s}>5.0 \mathrm{ps}^{-1} \text { at } 95 \% \mathrm{CL} \text {. }
$$

Using the error on $A, \sigma_{A}$, the sensitivity is found to be:

$$
\text { Sensitivity }=6.6 \mathrm{ps}^{-1} \text {. }
$$

The sensitivity would be $6.8 \mathrm{ps}^{-1}$ if the systematic error on the amplitude was neglected ${ }^{6}$.

${ }^{6}$ In Table 7 the results are given for the amplitude and its error as a function of $\Delta m_{s}$ after adjusting $f_{\mathrm{B}_{\mathrm{s}}}$ to the recently published value of 0.106 [13]. 


\section{Inclusive vertices and soft leptons}
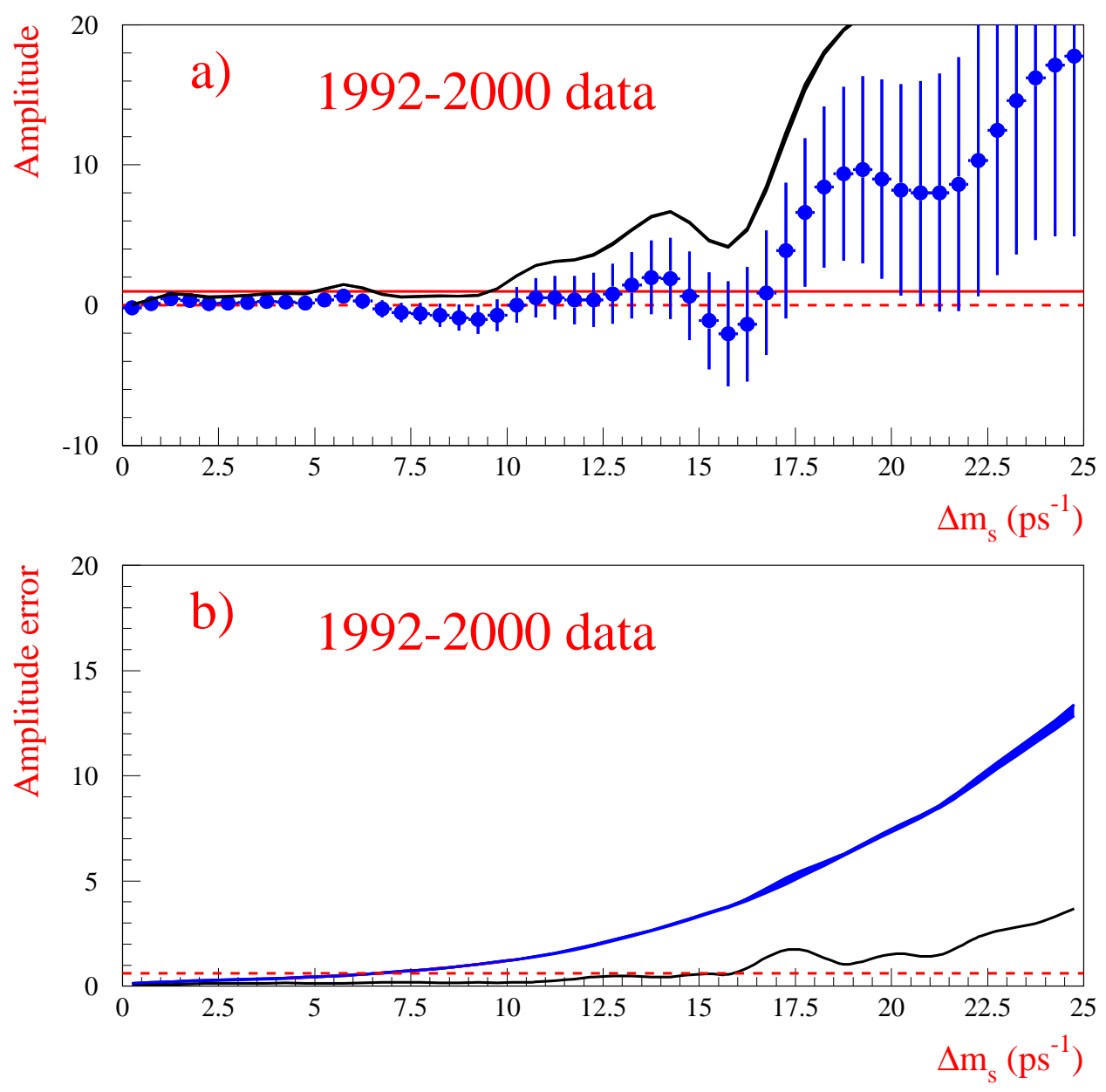

Figure 12: a) Fitted values of the oscillation amplitude $A$ as a function of $\Delta m_{s}$. The horizontal line corresponds to the value $A=1$. The black band is situated between the curves for $A+1.645 \sigma_{A_{\text {stat }}}$ and $A+1.645 \sigma_{A_{t o t}}$. b) The total amplitude error as a function of $\Delta m_{s}$. The upper band is situated between the statistical error $\left(\sigma_{A_{s t a t}}\right)$ and the total error $\left(\sigma_{A_{t o t}}\right)$. The lower curve shows the systematic error $\sigma_{A_{s y s}}$. The crossing point with the dashed line of the rising curve for the total error with $\sigma_{A}=1 / 1.645$ at $\Delta m_{s}=6.6 \mathrm{ps}^{-1}$ gives the sensitivity. 
Inclusive vertices and soft leptons

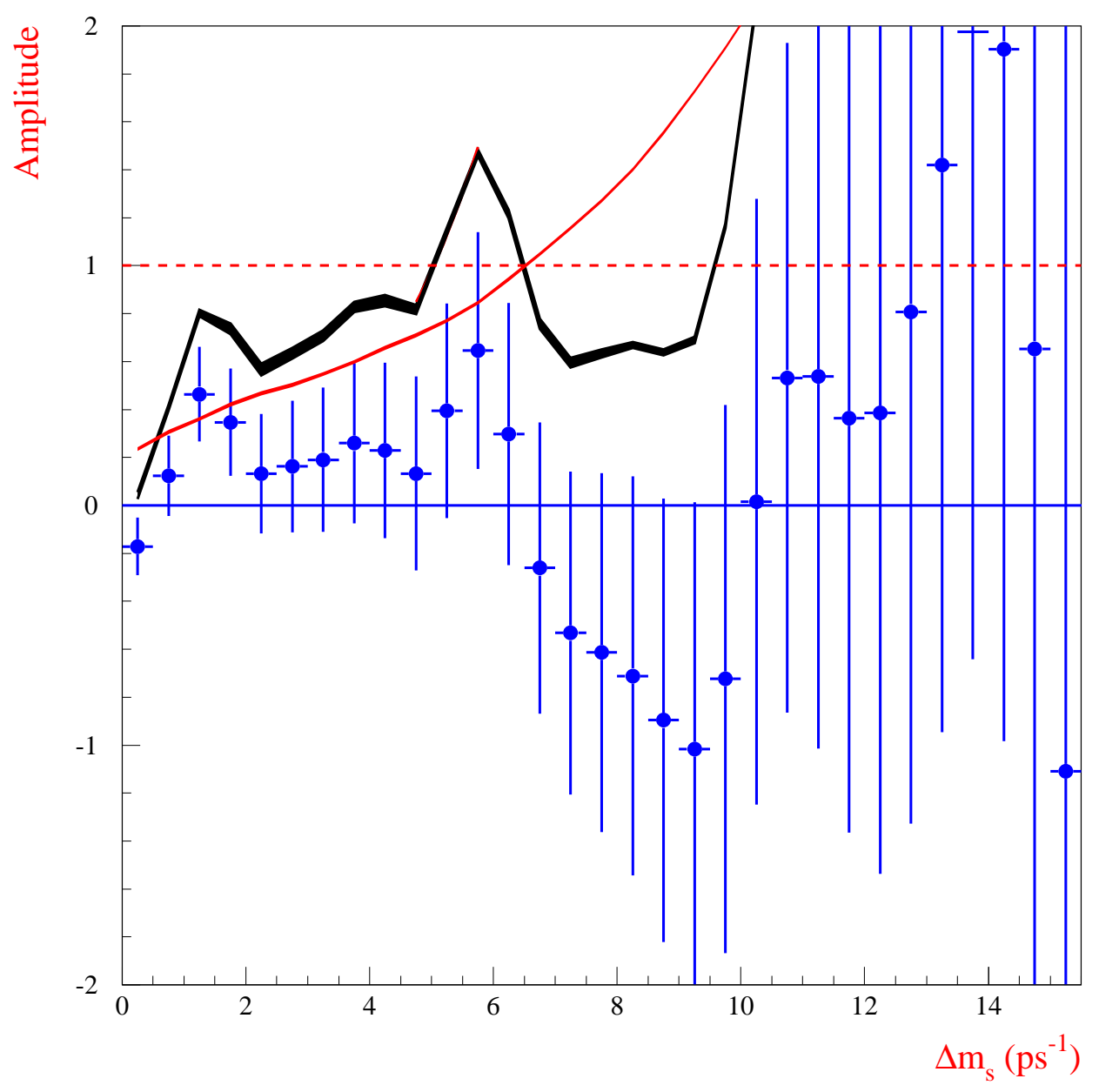

Figure 13: Fitted values of the oscillation amplitude $A$ as a function of $\Delta m_{s}$. The data are identical to those of Fig.12. The dashed horizontal line corresponds to $A=1$. The black band is situated between the curves for $A+1.645 \sigma_{A_{s t a t}}$ and $A+1.645 \sigma_{A_{t o t}}$. The smoothly rising curve corresponds to $1.645 \sigma_{A_{t o t}}$. The crossing point with $A=1$ at $\Delta m_{s}=6.6 \mathrm{ps}^{-1}$ gives the expected lower limit at 95\% CL. 


\begin{tabular}{|c|c|c|c|}
\hline$\Delta m_{s}\left(p s^{-1}\right)$ & $A$ & $\sigma_{A}($ stat $)$ & $\sigma_{A}($ total $)$ \\
\hline 0.25 & -0.17 & 0.11 & 0.13 \\
\hline 0.75 & 0.12 & 0.15 & 0.17 \\
\hline 1.25 & 0.46 & 0.18 & 0.20 \\
\hline 1.75 & 0.35 & 0.21 & 0.23 \\
\hline 2.25 & 0.13 & 0.23 & 0.26 \\
\hline 2.75 & 0.16 & 0.25 & 0.28 \\
\hline 3.25 & 0.19 & 0.28 & 0.31 \\
\hline 3.75 & 0.26 & 0.31 & 0.33 \\
\hline 4.25 & 0.23 & 0.34 & 0.37 \\
\hline 4.75 & 0.13 & 0.37 & 0.40 \\
\hline 5.25 & 0.39 & 0.41 & 0.43 \\
\hline 5.75 & 0.65 & 0.45 & 0.47 \\
\hline 6.25 & 0.30 & 0.50 & 0.53 \\
\hline 6.75 & -0.26 & 0.56 & 0.58 \\
\hline 7.25 & -0.53 & 0.62 & 0.65 \\
\hline 7.75 & -0.61 & 0.69 & 0.71 \\
\hline 8.25 & -0.71 & 0.76 & 0.78 \\
\hline 8.75 & -0.90 & 0.85 & 0.87 \\
\hline 9.25 & -1.02 & 0.95 & 0.96 \\
\hline 9.75 & -0.72 & 1.05 & 1.06 \\
\hline 10.25 & 0.02 & 1.16 & 1.17 \\
\hline 10.75 & 0.53 & 1.28 & 1.30 \\
\hline 11.25 & 0.54 & 1.43 & 1.45 \\
\hline 11.75 & 0.36 & 1.59 & 1.62 \\
\hline 12.25 & 0.39 & 1.77 & 1.82 \\
\hline 12.75 & 0.81 & 1.96 & 2.02 \\
\hline 13.25 & 1.42 & 2.17 & 2.23 \\
\hline 13.75 & 1.98 & 2.40 & 2.44 \\
\hline 14.25 & 1.90 & 2.65 & 2.69 \\
\hline 14.75 & 0.65 & 2.91 & 2.96 \\
\hline 15.25 & -1.11 & 3.17 & 3.22 \\
\hline 15.75 & -2.04 & 3.44 & 3.48 \\
\hline 16.25 & -1.36 & 3.74 & 3.84 \\
\hline 16.75 & 0.88 & 4.08 & 4.30 \\
\hline 17.25 & 3.90 & 4.45 & 4.77 \\
\hline 17.75 & 6.61 & 4.85 & 5.14 \\
\hline 18.25 & 8.43 & 5.27 & 5.44 \\
\hline 18.75 & 9.36 & 5.69 & 5.79 \\
\hline 19.25 & 9.66 & 6.12 & 6.23 \\
\hline 19.75 & 8.99 & 6.53 & 6.68 \\
\hline 20.25 & 8.22 & 6.92 & 7.09 \\
\hline 20.75 & 8.00 & 7.33 & 7.47 \\
\hline 21.25 & 8.02 & 7.79 & 7.93 \\
\hline 21.75 & 8.63 & 8.31 & 8.52 \\
\hline 22.25 & 10.32 & 8.89 & 9.19 \\
\hline 22.75 & 12.47 & 9.48 & 9.84 \\
\hline 23.25 & 14.58 & 10.05 & 10.43 \\
\hline 23.75 & 16.20 & 10.62 & 11.03 \\
\hline 24.25 & 17.11 & 11.21 & 11.68 \\
\hline 24.75 & 17.77 & 11.82 & 12.38 \\
\hline
\end{tabular}

Table 7: The amplitude and its statistical and systematic error as a function of $\Delta m_{s}$ after adjusting $f_{\mathrm{B}_{\mathrm{s}}}$ to the published value of 0.106 [13]. 


\section{A neural network analysis}

The inclusive $\mathrm{B}_{s}^{0}$ analysis described in this section was an attempt to optimize the statistical precision attainable in the high $\Delta m_{s}$ region. This analysis made extensive use of neural network techniques for tagging and vertex reconstruction, mostly based on the BSAURUS [12] package. Several neural networks were used on the event and track level. For optimal performance a good resolution on the proper time was required and this was achieved by keeping the energy and the vertex reconstruction separated in the analysis. The separated treatment of decay length and energy reconstruction led to a CPU intensive two-dimensional integration for each event. Only the best class of events (in terms of the decay length resolution) was used, to reach an optimal performance for high $\Delta m_{s}$ values. The restrictive cuts on quality and decay length resolution led to a sample of only $30 \mathrm{k}$ events for the data taken in 1994.

\subsection{Event selection}

Multihadronic $\mathrm{Z}^{0}$ events were selected requiring at least 5 reconstructed tracks and a total reconstructed energy larger than $12 \%$ of the centre-of-mass energy. The event was rejected if it had more than 3 jets or if the value of $\left|\cos \left(\theta_{\text {thrust }}\right)\right|$ was larger than 0.75. The cosine of the opening angle between the two most energetic jets was required to be less than -0.98 . Further, the value of the combined event b-tagging variable $x_{e v}$ as defined in ref. [5] had to be larger than 0.5. Events having an identified lepton with a transverse momentum larger than $1.2 \mathrm{GeV} / \mathrm{c}$ were removed. To obtain a homogeneous data set, it was required that both the liquid and gas radiators of the Barrel RICH were fully operational.

The same selection was applied to simulated $\mathrm{Z} \rightarrow q \bar{q}$ events using the JETSET 7.3 [6] generator.

Each event was split into hemispheres using the plane perpendicular to the thrust axis. A first estimate of the $\mathrm{B}$ candidate momentum vector was obtained by calculating the charged particles rapidities, with respect to the thrust axis, and by summing the momenta of those with rapidity $>1.6$. In each hemisphere a secondary vertex was fitted using the tracks with vertex detector hits from high rapidity charged particles. The secondary vertex fit was performed in three dimensions using as a constraint the direction of the B candidate momentum vector. The result of the vertex fit was used as an input to a Neural Network, the so-called TrackNet, that distinguishes between a fragmentation track and a track originating from a weakly decaying $\mathrm{B}$ hadron. In the final stage of the fit, the TrackNet output was used to add candidate tracks to the secondary vertex and the fit was redone.

Finally, a hemisphere was rejected if the secondary vertex fit did not converge.

\subsection{Flavour tagging}

The tagging of the quark flavour at production and decay times is necessary to distinguish mixed from unmixed $\mathrm{B}_{s}^{0}$ mesons. Only the opposite hemisphere was used for the production tag to reduce correlations between the production and decay tags. The decay tag was based on track-by-track flavour nets, which were later combined using a likelihood ratio to tag the presence of a $\mathrm{B}$ or $\overline{\mathrm{B}}$ meson at decay time in each hemisphere. For the production tag a dedicated neural network was used. 


\subsection{The track-by-track flavour nets}

Eight different networks were trained corresponding to a production and a decay flavour network for each of the four B hadron types. The aim of each network was to exploit, track-by-track, the correlation between the charge of a single track and the b quark charge. This approach is motivated by the different decay chains of the various types of $\mathrm{B}$ hadrons where, for example, the 'charge' of the $D$ meson determines the b quark charge.

The discriminating input variables are: particle identification (e.g. kaon, proton and lepton probabilities), B-D vertex separation based on a network trying to discriminate between tracks originating from the weakly decaying $B$ hadron and those from the subsequent cascade $D$ meson decay, the momenta in the $\mathrm{B}$ rest frame and variables related to tracking quality. The track decay flavour nets use 21 input variables in total, while the track production flavour nets have 18 input variables; essentially the same input variables without the lepton identification and the B-D net variables.

To obtain a flavour tag in a given hemisphere the individual track probabilities $P(\text { track })_{i}^{j}\left(i=\mathrm{B}_{\mathrm{u}}, \mathrm{B}_{\mathrm{d}}, \mathrm{B}_{\mathrm{s}}, \mathrm{B}_{\text {baryon }}\right.$ and $j=$ production or decay) coming from the different networks were combined in the following way,

$$
P(\text { hem })_{i}^{j}=\sum_{\text {tracks }} q(\text { track }) \log \frac{1+P(\text { track })_{i}^{j}}{1-P(\text { track })_{i}^{j}},
$$

where $q($ track $)$ is the charge. For the production flavour tag, tracks with TrackNet values less than 0.5 are selected, while for the decay flavour tag, tracks must have a TrackNet value above 0.5 .

\subsection{The $\mathrm{B}_{s}^{0}$ production and decay flavour tag}

The production flavour net was constructed using all the information available in the hemisphere, i.e. the fragmentation and decay flavour probabilities $P(h e m)_{\mathrm{B}_{\mathrm{u}}, \mathrm{B}_{\mathrm{d}}, \mathrm{B}_{\mathrm{s}}, \mathrm{B}_{\text {baryon }} \text {, }}^{\text {rod, }}$, and the quality of the information for the selected hemisphere. More details on the flavour networks and on the flavour tag can be found in [12].

In Figure 14a the probability distribution for the production tag for 1994 data and

simulation is shown. The grey lines indicate the distributions for $\mathrm{b}$ and $\overline{\mathrm{b}}$ quarks. The achieved tagging purity on simulation is $71 \%$ at $100 \%$ efficiency.

For the $\mathrm{B}_{s}^{0}$ decay flavour tag, the probability $P(\text { hem })_{B_{s}^{0}}^{\text {decay }}$ was used. In Figure $14 \mathrm{~b}$ the $\mathrm{B}_{s}^{0}$ decay flavour probability distributions for 1994 data and simulation are shown. The tagging purity on simulation was $62 \%$ at $100 \%$ efficiency. The contributions from light and charm quarks are very small due to the high b purity of the sample of $98.3 \%$.

\subsection{Energy reconstruction}

To determine the proper time, a precise estimate of the energy of the decaying $\mathrm{B}$ hadron is needed. The starting point was a raw estimate of the B energy $E_{\text {raw }}$ and mass $m_{\text {raw }}$. These quantities were determined by weighting (with a sigmoid threshold function) the momentum and energy components of the charged particles by the TrackNet output value and the neutral particles by their rapidity. For three-jet events only the rapidity was used as a weight. In this way particles coming from the decaying B hadron receive a higher weight. 

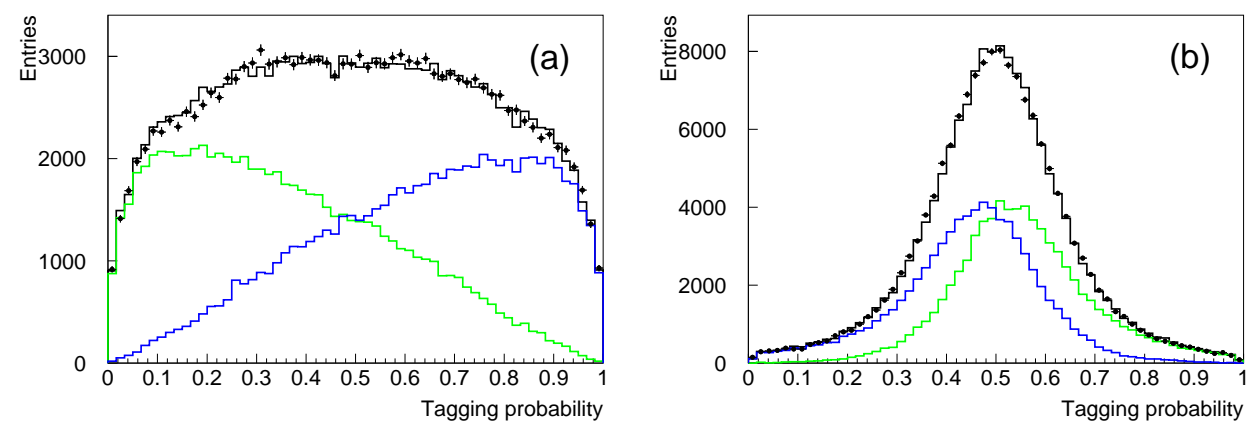

Figure 14: The production (a) and decay (b) tag distributions for 1994 data and simulation. The distributions for $\mathrm{b}$ and $\overline{\mathrm{b}}$ are indicated in light and dark grey. The decay tag is optimized for $\mathrm{B}_{\mathrm{s}}$ and $\overline{\mathrm{B}_{\mathrm{s}}}$ particles.

The raw energy was corrected as a function of $m_{\text {raw }}$ and of the fraction of the energy in the hemisphere, $x_{h}$, to obtain an improved estimate of the energy. This was done in the following way. The simulated data were divided into several samples according to the measured ratio $x_{h}$ and for each of these samples the $\Delta E$, defined as the true energy minus the raw energy, was plotted as function of $m_{\text {raw }}$. The median values of $\Delta E$ in each bin of $m_{\text {raw }}$ were calculated and the $m_{\text {raw }}$ dependence was fitted by a third order polynomial:

$$
\Delta E\left(m_{\text {raw }}, x_{h}\right)=a+b\left(m_{\text {raw }}-\left\langle m_{\text {raw }}\right\rangle\right)+c\left(m_{\text {raw }}-\left\langle m_{\text {raw }}\right\rangle\right)^{2}+d\left(m_{\text {raw }}-\left\langle m_{\text {raw }}\right\rangle\right)^{3} .
$$

The four parameters $a, b, c, d$ in each $x_{h}$ bin were then studied as functions of $x_{h}$ and parametrized with third and second-order polynomials. In this way a smooth correction function was obtained.

This procedure led to an estimate of the B hadron energy. Studies on simulated $\mathrm{B}$ events showed a large correlation between the number of tracks and the $\mathrm{B}$ energy resolution. For this reason, the number of tracks in the hemisphere was chosen to define different resolution classes. In total 16 different classes were defined, starting with 2 tracks per hemisphere in class one and ending with 17 and more tracks in class 16 . The central Gaussian of a double Gaussian fit to the B energy resolution varies from $4 \%$ in the best class to $15 \%$ in the worst.

\subsection{Decay length reconstruction}

Starting from the secondary vertex algorithm, described in section 3.1, an optimized algorithm was developed with the aim of improving the decay length resolution and of minimizing the forward bias resulting from the inclusion of tracks from the cascade $D$ decay vertex in the B decay vertex reconstruction. Based on the output of the B-D net, a so-called 'Stripping' algorithm was developed.

For the 'Stripping' algorithm candidate tracks were selected if they had a TrackNet output larger than 0.5 and a B-D net output value less than 0.45 . The B-D net cut value corresponds to an efficiency of $50 \%$ for selecting a track from a weakly decaying B hadron at a purity of $75 \%$. A secondary vertex fit was performed if two or more tracks were selected. If the fit failed to converge within the algorithm criteria and more than two tracks were selected, the track with highest $\chi^{2}$ contribution was removed and the fit 


\begin{tabular}{|c|c||c|c|}
\hline particle & decay tag $\alpha_{D}$ & particle & production tag $\alpha_{P}$ \\
\hline $\mathrm{B}_{s}^{0}$ & 1 & b quarks & 0.94 \\
$\mathrm{~B}_{d}$ & 1.08 & c quarks & 0.56 \\
$\mathrm{~B}_{u}$ & 1.15 & uds quarks & 0.84 \\
$\mathrm{~B}_{\text {baryon }}$ & 0.93 & & \\
c quarks & 1.05 & & \\
uds quarks & 0.08 & & \\
\hline
\end{tabular}

Table 8: The $\alpha$ parameters for the decay and production tag for the different particles as obtained from the 1994 simulation

was repeated. This procedure was done iteratively until convergence was reached or two tracks were left. Finally, the direction of the B, as estimated by the B energy algorithm, was used as a constraint. The overall efficiency to find a vertex was about $50 \%$.

Events with a very good decay length resolution were selected by requiring that the expected error on the decay length was smaller than $200 \mu \mathrm{m}$.

Because of cuts on the TrackNet output, on the B-D output and on the expected decay length error, less events will be reconstructed at small decay length. Therefore an acceptance function depending on the true B decay length was calculated using the simulation.

After having applied these cuts, 30k hemispheres were selected in the 1994 data sample. The b purity of the sample was estimated from simulated events to be $98.3 \%$.

\subsection{The likelihood fit}

In the fitting program, the like- and unlike-sign events were separated in the same way as described in section 2.5 of the previous analysis and the same expressions for like- and unlike-sign probabilities were used.

A difference from the previous analysis was the treatment of the resolution functions $\mathcal{R}\left(l_{\text {rec }}-l_{\text {true }}, l_{\text {true }}\right)$ and $\mathcal{R}\left(\left(p_{\text {rec }}-p_{\text {true }}\right) / p_{\text {true }}\right)$, which were kept separated. As a parameterization for the decay length $l$ two asymmetric Gaussian distributions were chosen, while for the momentum reconstruction two symmetric Gaussian distributions were used. The probability for a $\mathrm{B}$ event to be observed at a proper time $P\left(t_{\text {rec }}\right)$ is a convolution over an exponential B decay distribution, an acceptance function $A(l, p)$, the true $\mathrm{B}$ hadron momentum distribution $F(p)$ and the resolution functions $\mathcal{R}_{l}$ and $\mathcal{R}_{p}$, all four taken from simulation:

$$
\mathcal{P}_{b}\left(t_{\text {rec }}\right)=\int_{l=0}^{\infty} \int_{p=0}^{\infty} A(l, p) F(p) \mathcal{R}_{l}\left(l_{\text {rec }}-l, l\right) \mathcal{R}_{p}\left(\left(p_{\text {rec }}-p\right) / p\right) \frac{e^{-l m /(\tau p)}}{\tau} d l d p,
$$

were $\tau$ denotes the B lifetime and Eq. (8) was used to calculate the proper time.

\subsection{Modelling simulation and data}

As explained in section 2.6 it is important to model precisely the tagging purities. In this analysis the raw purities were modified using a parameter $\alpha$ as defined in Eq. (17). The decay and production tag parameters for the different particles were obtained from simulation, and are listed in Table 8. 

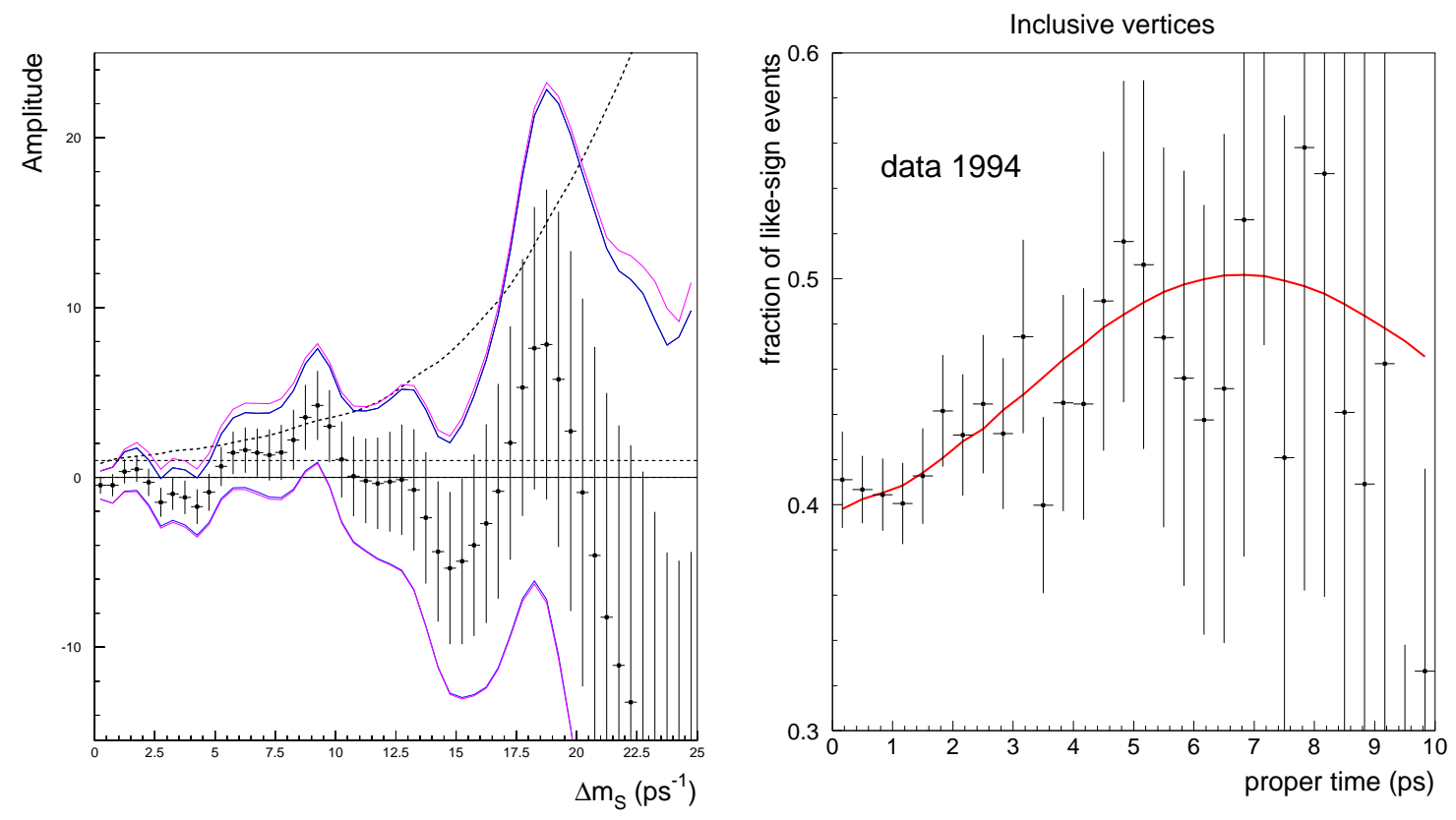

Figure 15: In the left plot the fitted $\mathrm{B}_{s}^{0}$ oscillation amplitude for the NN analysis is shown as a function of $\Delta m_{s}$ as points and error bars. The continuous (dotted) lines correspond

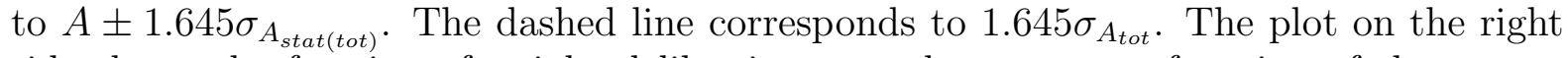
side shows the fraction of weighted like-sign tagged events as a function of the proper time. The data are shown as points with error bars, the parametrization is given as a solid line.

For the real data, the correction factor $C$, defined in Eq. (19), was determined from the fraction of like-sign events, using the same method as was discussed in section 2.6. Two correction factors were needed, one for $\mathrm{B}_{u}$ mesons and one for the other $\mathrm{B}$ mesons. Their values were $C_{\mathrm{B}_{\mathrm{u}}}=0.53$ and $C=0.81$.

Using the amplitude method [11] the result shown in Figure 15a was obtained. A limit on $\Delta m_{s}$ was not extracted as the analysis was optimized for high values of $\Delta m_{s}$. Figure $15 \mathrm{~b}$ shows the agreement between the data and the description by the fitting programme.

Systematic uncertainties have been evaluated by varying a single parameter at a time (e.g $f_{\mathrm{B}_{\mathrm{s}}}$ ) and redoing the full amplitude fit. The systematic error was then calculated as defined in Eq. (25). The same parameters as described in section 2.8 were varied and the systematic error was determined to be at most $25 \%$ of the statistical error.

The error on the fitted $\mathrm{B}_{s}^{0}$ amplitude at $\Delta m_{s}$ of 15 and $20 \mathrm{ps}^{-1}$ gives respectively 5.1 and 11.8 for this analysis using only 1994 data. This can be compared with the values of 5.0 and 10.9 obtained with the previous analysis using only the 1994 data sample. The results of the neural network analysis optimized for high values of $\Delta m_{s}$ are compatible with the results for the 1992-2000 data shown in section 2.8. No attempt is made to combine the results. 


\section{Conclusion}

Using a total sample of $770 \mathrm{k}$ events - of which $155 \mathrm{k}$ events contain a soft lepton the mass difference between the two physical states in the $\mathrm{B}_{d}^{0}-\overline{\mathrm{B}_{d}^{0}}$ system was measured to be:

$$
\Delta m_{d}=(0.531 \pm 0.025(\text { stat. }) \pm 0.007(\text { syst. })) \mathrm{ps}^{-1} .
$$

The following limit on the width difference between the two states was obtained:

$$
\left|\Delta \Gamma_{\mathrm{B}_{\mathrm{d}}}\right| / \Gamma_{\mathrm{B}_{\mathrm{d}}}<0.18 \text { at } 95 \% \mathrm{CL} \text {. }
$$

As no evidence for $\mathrm{B}_{s}^{0}-\overline{\mathrm{B}_{s}^{0}}$ oscillations was found, a limit on the mass difference of the two physical states was given:

$$
\Delta m_{s}>5.0 \mathrm{ps}^{-1} \text { at } 95 \% \mathrm{CL}
$$

with a sensitivity equal to $6.6 \mathrm{ps}^{-1}$.

These results are compatible with a neural network analysis optimized for high values of $\Delta m_{s}$.

\section{Acknowledgements}

We are greatly indebted to our technical collaborators, to the members of the CERNSL Division for the excellent performance of the LEP collider, and to the funding agencies for their support in building and operating the DELPHI detector.

We acknowledge in particular the support of Austrian Federal Ministry of Education, Science and Culture, GZ 616.364/2-III/2a/98, FNRS-FWO, Flanders Institute to encourage scientific and technological research in the industry (IWT), Belgium,

FINEP, CNPq, CAPES, FUJB and FAPERJ, Brazil,

Czech Ministry of Industry and Trade, GA CR 202/99/1362,

Commission of the European Communities (DG XII),

Direction des Sciences de la Matière, CEA, France,

Bundesministerium für Bildung, Wissenschaft, Forschung und Technologie, Germany,

General Secretariat for Research and Technology, Greece,

National Science Foundation (NWO) and Foundation for Research on Matter (FOM), The Netherlands,

Norwegian Research Council,

State Committee for Scientific Research, Poland, SPUB-M/CERN/PO3/DZ296/2000,

SPUB-M/CERN/PO3/DZ297/2000, 2P03B 10419 and 2P03B 69 23(2002-2004)

JNICT-Junta Nacional de Investigação Científica e Tecnológica, Portugal,

Vedecka grantova agentura MS SR, Slovakia, Nr. 95/5195/134,

Ministry of Science and Technology of the Republic of Slovenia,

CICYT, Spain, AEN99-0950 and AEN99-0761,

The Swedish Natural Science Research Council,

Particle Physics and Astronomy Research Council, UK,

Department of Energy, USA, DE-FG02-01ER41155. 


\section{References}

[1] G. Altarelli and P.J. Franzini, Zeit. Phys C37 (1988) 271.

P.J. Franzini, Phys. Rep. 173 (1989) 1.

[2] M. Ciuchini et al., JHEP 0107:013, 2001 hep-ph/0012308.

[3] L. Wolfenstein, Phys. Rev Lett. 51 (1983) 1945.

[4] DELPHI Coll., P. Abreu et al., Eur. Phys. J C18 (2000) 229,

DELPHI Coll., P. Abreu et al., Eur. Phys. J. C16 (2000) 555,

DELPHI Coll., P. Abreu et al., Phys. Lett. B414 (1997) 382.

[5] G. V. Borisov and C. Mariotti, Nucl. Instr. and Meth. A372 (1996) 181, G. V. Borisov Combined b-tagging, DELPHI Note, PHYS 716-94 (1997).

[6] T. Sjöstrand, PYTHIA 5.7 and JETSET 7.4, Computer Physics Commun. 82 (1994) 74.

[7] DELPHI Coll., P. Aarnio et al., Nucl. Inst. Meth. A303 (1991) 233,

DELPHI Coll., P. Abreu et al., Nucl. Inst. Meth. A378 (1996) 57.

[8] DELPHI Coll., P. Abreu et al, Zeit. Phys. C71 (1996) 11.

[9] DELPHI Coll., "DELSIM Reference Manual", DELPHI 87-97 PROG-100.

[10] ALEPH, CDF, DELPHI, L3, OPAL, SLD Coll., "Combined results on b-hadron production rates, lifetimes, oscillations and semileptonic decays", CERN-EP-2000096.

Recent updates http://lepbosc.web.cern.ch/LEPBOSC/ combined_results/.

[11] H. G. Moser and A. Roussarie, Nucl. Instr. and Meth. A384 (1997) 491.

[12] Z. Albrecht et al., The BSAURUS package, hep-ex/0102001.

[13] K. Hagiwara et al., Phys. Rev. D66, 010001 (2002). 